\title{
Excited state intramolecular charge transfer reaction in non-aqueous reverse micelles: Effects of solvent confinement and electrolyte concentration ${ }^{\#}$
}

\author{
TUHIN PRADHAN, HARUN AL RASID GAZI, BISWAJIT GUCHHAIT and RANJIT \\ BISWAS* \\ Department of Chemical, Biological and Macromolecular Sciences, and Unit for Nanoscience and Technology, \\ S. N. Bose National Centre for Basic Sciences, JD Block, Sector III, Salt Lake, Kolkata 700 098, India \\ e-mail: ranjit@bose.res.in
}

MS received 10 February 2011; revised 31 May 2011; accepted 6 June 2011

\begin{abstract}
Steady state and time resolved fluorescence emission spectroscopy have been employed to investigate the effects of solvent confinement and electrolyte concentration on excited state intramolecular charge transfer (ICT) reaction in 4-(1-pyrrolidinyl) benzonitrile (P5C), 4-(1-piperidinyl) benzonitrile (P6C), and 4-(1-morpholenyl) benzonitrile (M6C) in AOT/n-heptane/acetonitrile and AOT/n-heptane/methanol reverse micelles. Dramatic confinement effects have been revealed via a huge reduction (factor ranging between 100 and 20) over bulk values of both equilibrium and reaction rate constants. A strong dependence on the size of the confinement $\left(W_{s}\right)$ of these quantities has also been observed. $W_{s}$ dependent average static dielectric constant, viscosity and solvation time-scale have been determined. Estimated dielectric constants for confined methanol and acetonitrile show a decrease from the respective bulk values by a factor of 3-5 and viscosities increased by a factor of 2 at the highest $W_{s}$ considered. Addition of electrolyte at $W_{s}=5$ for acetonitrile is found to produce a linear increase of confined solvent viscosity but leads to a non-monotonic electrolyte concentration dependence of average solvation time. Reaction rate constant is found to decrease linearly with electrolyte concentration for P5C and P6C but non-monotonically for $\mathrm{M} 6 \mathrm{C}$, the highest decrease for all the molecules being $\sim 20 \%$ over the value in the absence of added electrolyte in the solvent pool. The observed huge reduction in reaction rate constant is attributed to the effects of decreased solution polarity, enhanced viscosity and slowed-down solvent reorganization of the solvent under confinement in these non-aqueous reverse micelles.
\end{abstract}

Keywords. Intramolecular charge transfer reaction; non-aqueous reverse micelles; confinement effects; static and dynamic solvent control.

\section{Introduction}

Reverse micelles are formed via self aggregation in a ternary mixture of surfactant, polar and non-polar solvents. ${ }^{1-27}$ A subtle balance between dispersive and electrostatic interactions is the key for the multiple components to remain in a single solution phase. The most commonly used surfactant is sodium bis (2ethylhexyl)-sulfosuccinate (AOT) which possesses charged (anionic) head group and forms both aqueous and non-aqueous reverse micelles in presence of a nonpolar solvent (usually isooctane or heptane). The polar solvents trapped inside reverse micelles behave differently from the pure solvents because geometric

\footnotetext{
*For correspondence

${ }^{\#}$ The paper is dedicated to the fond memory of Dr. Suhrita Bagchi.
}

confinement substantially modifies many bulk solvent properties. Eventhough water has traditionally been the most common polar solvent component, a few nonaqueous polar solvents have also been used to form reverse micelles. Use of these non-aqueous polar solvents has been motivated partly by the desire to investigate whether aqueous and non-aqueous reverse micelles behave similarly in solvent encapsulation and partly to employ the confined non-aqueous media as microreactors for certain reactions. ${ }^{28-31}$

Recently, AOT/isooctane (or heptane)/polar solvent reverse micelles with methanol and acetonitrile as polar liquids have been characterized by dynamic light scattering (DLS) experiments and ultraviolet-visible (UV-vis) absorption spectroscopy. ${ }^{19}$ An important issue here is the partial solubility of methanol and acetonitrile in bulk nonpolar solvent (heptane or isooctane) 
as this allows dispersion of polar solvent into the nonpolar phase and thus leading to less swelling of reverse micelle droplets at higher loadings of polar solvents. A correlation between the size of these non-aqueous reverse micelles and ratio $\left(\mathrm{W}_{\mathrm{s}}\right)$ between concentrations of polar solvent and surfactant $\left(\mathrm{W}_{\mathrm{s}}=\right.$ [polar solvent]/ [AOT]) attempted in this study ${ }^{19}$ reveals that while methanol reverse micelles registers an over-all increase in diameter from $\sim 3 \mathrm{~nm}$ to $\sim 5 \mathrm{~nm}$ in changing $\mathrm{W}_{\mathrm{s}}$ from $\sim 1$ to 10 , the size remains almost unaltered for acetonitrile between $\mathrm{W}_{\mathrm{s}}$ values 1 and 5. Absorption data using a dye suggests that, in the above narrow size range, the polar solvents are confined in the cavity formed via the aggregation of surfactant head-groups. However, polar solvent partitioning into the nonpolar phase has been reported at higher values of $\mathrm{W}_{\mathrm{s}}$. Further studies with methanol reverse micelles using Fourier transform infrared (FTIR) spectroscopy ${ }^{32,33}$ indicate the presence of broadly two kinds of methanol moleculesbound and free. While the bound molecules are considered as those found at and within the ionic layer constituted by $\mathrm{Na}^{+}$and $-\mathrm{SO}_{3}^{-}$ions, the free ones (bulk type) are residing either at the centre of the cavity or dispersed out into the non-polar phase and existing as monomeric or other aggregated forms. Interestingly, while a few investigators ${ }^{19,32}$ interpret their spectroscopic results in terms of change in size of reverse micelle with $\mathrm{W}_{\mathrm{s}}$, others ${ }^{33}$ attribute the observed dependence to the change in shape (for example, spherical to ellipsoid) only. Even though similar studies with acetonitrile reverse micelles have not been carried out yet, dynamic fluorescence Stokes' shift measurements with several coumarin dyes have repeatedly indicated $\mathrm{W}_{\mathrm{s}}$ insensitivity for acetonitrile reverse micelles but moderate dependence for above. ${ }^{19}$

Addition of electrolyte in confined pool, on the other hand, may affect the environment in many ways. First, phase transition (structural) may occur from oilin-water (o/w, Winsor I system) type to water-in-oil (w/o, Winsor II system) type via an intermediate phase (Winsor III system). ${ }^{39-41}$ Therefore, addition of electrolyte in reverse micelles may be used as an experimental control variable where structural transition can be engineered and used for desired product formation. ${ }^{21}$ Second, addition of electrolytes affects the existing electrostatic interactions in a confined pool by supplying additional ions and ion-pairs in the medium. ${ }^{42}$ This leads to the modification in droplet size and its ability to accommodate the host particles. In addition, the viscosity and average polarity of the confined medium are expected to be different in presence of added salts and hence environment response time scale will be altered. This may impart significant effects on a reaction occurring in such micro-environment in presence of electrolyte.

Recently, photo-induced intramolecular charge transfer (ICT) reaction has been studied in aqueous and several non-aqueous AOT/heptane reverse micelles where the importance of the reverse micelle interface to control an ICT process are stressed. ${ }^{22(a), 37,43}$ Another recent study ${ }^{10}$ of ICT reaction occurring in aqueous reverse micelles has revealed more than an order of magnitude slowing down of the reaction rate constant in aqueous pool compared to that in a bulk solvent of comparable polarity (in terms of static dielectric constant, $\left.\varepsilon_{0}\right)$ and viscosity. The role of sluggish medium dynamics for lengthening the reaction time constant has been stressed in that work ${ }^{10}$ for the first time. However, whether such an effect is also operative for reverse micelles with non-aqueous polar solvents of much lower $\varepsilon_{0}$, has not yet been studied. Furthermore, effects of electrolyte on an ICT reaction in confined pool needs to be investigated in order to understand the confinement-induced modification of the electrolyteconcentration dependent reaction rate constant of a TICT reaction already measured in bulk electrolyte solutions. ${ }^{44-46}$ Here, we report such a study where photo-induced intramolecular charge transfer reaction has been investigated in AOT/heptane non-aqueous reverse micelles at different $W_{s}$ values, and also in presence of electrolytes in confined solvent at a fixed $\mathrm{W}_{\mathrm{s}}$. The non-aqueous polar solvents considered in this study are methanol and acetonitrile which are different in association character but of comparable polarities ( $\varepsilon_{0}$ values are 32.66 and 35.94 respectively). ${ }^{47}$ In addition, reverse micelles with these polar solvents are well-characterized. ${ }^{19,32,33}$ Therefore, use of these two solvents will assist in unravelling the role of solvent association character on ICT reaction in confinement.

Three TICT molecules, 4-(1-pyrrolidinyl) benzonitrile (P5C), 4-(1-piperidinyl) benzonitrile (P6C), and 4-(1-morpholenyl) benzonitrile (M6C), are used for the proposed study. The chemical structures of these molecules are shown in scheme 1. Note that earlier study with these molecules in bulk solvents indicate a reaction activation barrier of $\sim 3-4 k_{B} T$ which is not large enough to inhibit breaking down of conventional kinetics observed in the case of high barrier reaction. ${ }^{48,49}$ Consequently, it would be interesting if one encounters non-exponential reaction kinetics for these molecules in these reverse micelles, as observed earlier for several bulk polar solvents. ${ }^{48}$ Moreover, study with these three TICT molecules is required to establish the generality of the results for a given family of reactant 
molecules in these complex environments. As the polarity of a reaction medium plays a very important role in determining both the yield and the rate of ICT reaction in these molecules, ${ }^{50-54}$ we have made a continuum model estimate of the average polarity (in terms of static dielectric constant, $\varepsilon_{0}$ ) of the confined polar solvent pool by following the steady state fluorescence emission of a non-reactive polarity probe, coumarin 153
(C153, also shown in scheme 1). Note that extensive spectroscopic investigation with $\mathrm{C} 153$ in a wide range of room temperature liquids has revealed that this probe does not participate in specific solute-solvent (such as H-bondng) interaction. ${ }^{55-57}$ Solution dynamics inside the solvent pool in the reverse micelles has thus been investigated by monitoring the time-evolution of the fluorescence emission of the same probe molecule (C153).
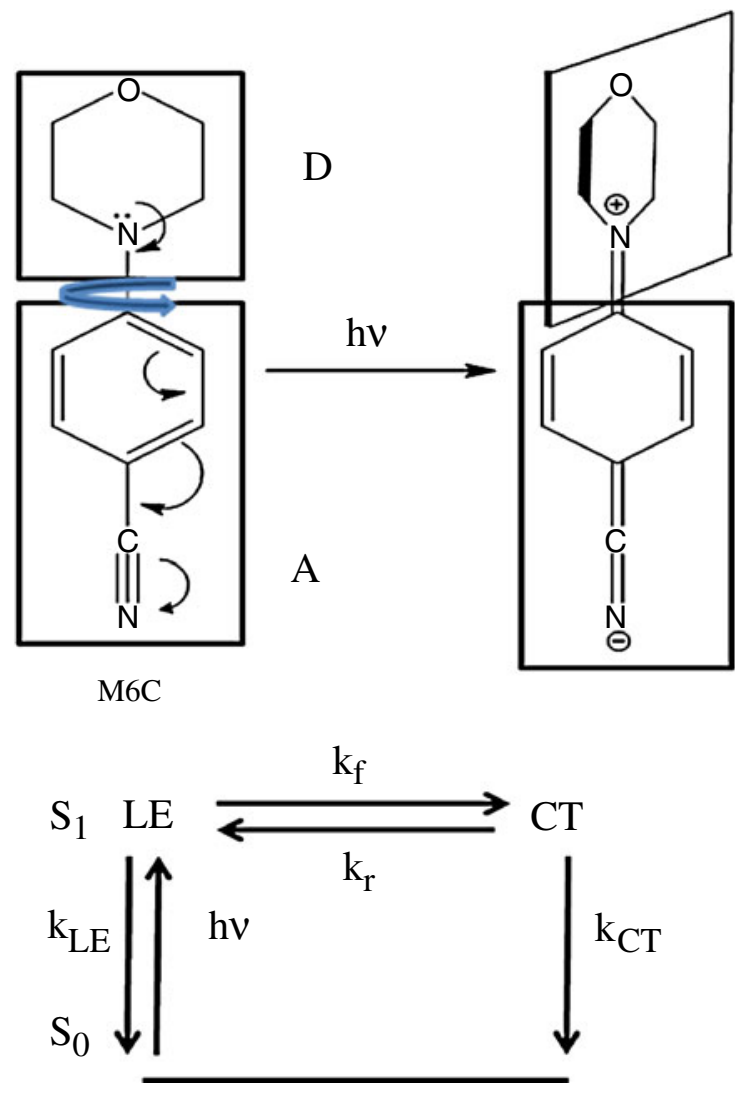<smiles>N#Cc1ccc(N2CCCC2)cc1</smiles>

P5C<smiles>N#Cc1ccc(N2CCCCC2)cc1</smiles>

P6C<smiles>O=c1cc(C(F)(F)F)c2cc3c4c(c2o1)CCCN4CCC3</smiles>

$\mathrm{C} 153$

Scheme 1. Pictorial representations of photo-excited intramolecular charge transfer reaction, reaction kinetics and chemical structures of solutes used. 
Since solvation time-scale is known to influence reaction rate, ${ }^{58,59}$ estimation of the former is crucial for interpreting the experimental results. We shall shortly see that ICT reaction in these molecules also thought to involve twisting ${ }^{48-51}$ and thus expected to couple to the local 'stiffness' of the medium. Fluorescence anisotropy measurements have been carried out to estimate such stiffness or local viscosity experienced by the twisting mode while reacting inside a confined pool.

We have used the twisted intramolecular charge transfer (TICT) model ${ }^{50,51,60-62}$ to explain the decay kinetics of these ICT molecules, although alternative arguments can also be employed. ${ }^{63-66}$ Since the mechanism of TICT reaction is already discussed elsewhere, ${ }^{48-51}$ we describe the mechanism very briefly here with the help of scheme 1. Upon photo-excitation, locally excited (LE) state is produced and converted to the relatively more polar charge transfer (CT) state with a forward reaction rate constant, $\mathrm{k}_{\mathrm{f}}$. There may be a barrier involved with the excited state $\left(\mathrm{S}_{1}\right)$ conversion from LE state to the CT state. ${ }^{48}$ Subsequently, the CT state can either regenerate the LE state by participating in the reverse reaction with a rate constant, $\mathrm{k}_{\mathrm{r}}$, or, decay to the ground state $\left(\mathrm{S}_{0}\right)$ with net (radiative + non-radiative) rate constant, $\mathrm{k}_{\mathrm{CT}}$. The photo-prepared LE state is depopulated to the ground state with the net rate constant, $\mathrm{k}_{\mathrm{LE}}$. In such a model, $\mathrm{LE} \rightarrow \mathrm{CT}$ inter-conversion reaction can be described by a two-state scheme. Additionally, it has been found that the inter-conversion reaction rate is generally faster than the LE and CT decay rates. ${ }^{48}$ In such a situation and in the absence of solvation dynamics on the reaction time scale, it has been shown that the time evolution of the LE or CT population could be described by a bi-exponential function of time. $^{48}$

\section{Experimental details}

\subsection{Sample preparation}

P5C, P6C and M6C were synthesized by following literature method. ${ }^{61,62}$ and re-crystallized twice using cyclohexane (Merck, Germany) as solvent. The absence of impurity in these synthesized compounds was ensured via thin layer chromatography (TLC) and monitoring the excitation wavelength dependence of fluorescence emission in several bulk polar and non-polar solvents. Methanol, acetonitrile and $\mathrm{LiClO}_{4}$ were used as received (highest grade, Aldrich). Bis(2-ethylhexyl) sodium sulfosuccinate (AOT) ( $\geq 98 \%$, Fluka and Sigma, $\geq 99 \%$ ) was used after vacuum drying for $24 \mathrm{~h}$. Spectroscopic grade n-heptane (Merck, $\geq 99.5 \%$ ) were used after drying over molecular sieves and filtration. Measured amount of solid AOT was dissolved in n-heptane to make solutions. Subsequently, polar solvent or solution of polar solvent with known electrolyte concentration was added to the solution to obtain appropriate $W_{s}$. The concentration of AOT in all reverse micelles was maintained at $0.1 \mathrm{M}$. The $W_{s}$ values were restricted up to the limit at which no phase separation was observed and also earlier reports ${ }^{19,32-38}$ of polar solvents being mostly inside the cavity. The highest concentration of added electrolyte $\left(\mathrm{LiClO}_{4}\right)$ was restricted at $0.9 \mathrm{M}$ because of instability of reverse micelles at electrolyte concentration larger than $0.9 \mathrm{M}$.

\subsection{Steady state measurements and estimation of spectral properties}

Steady state absorption and emission spectra were recorded by using respectively an absorption spectrophotometer (Shimadzu, UV - 2450) and a fluorimeter (SPEX fluoromax - 3, Jobin-Yvon, Horiba). Fluorescence spectra were collected after adjusting the absorbance of the sample to $\sim 0.1$ (concentration of ICT molecules in solution $<10^{-5}$ moles-litre ${ }^{-1}$ ). Solutions were taken in an optically transparent quartz cuvette with $1 \mathrm{~cm}$ optical path length before measurements. For a given sample, the peak wavelength $(\lambda)$ of the absorption spectrum was used as excitation wavelength for the corresponding emission scan. The fluorescence spectra were corrected for the wavelength dependence of the sensitivity of the apparatus. Solvent background was subtracted from the spectrum of samples. The spectrum was then converted properly to the frequency plane before calculating several steady state properties. Dry argon gas was passed into a few samples and emission spectra were collected. These runs showed very little or no effects on the spectra of charge transfer molecules in solutions and also on the decay kinetics.

LE and CT bands were obtained after deconvolution of the full emission spectrum of the TICT molecules into two fragments by shifting and broadening the reference emission spectrum of the corresponding TICT molecules in a least interacting solvent, which was chosen to be perfluorohexane. ${ }^{48}$ The linewidth of the individual bands (LE and CT) were determined by convoluting the reference emission with a Gaussian line broadening function (inhomogeneous solvent broadening assumed). A given experimental emission spectrum was then least-squared fit to the model spectrum consisting of two bands derived from the shifted and 
broadened reference emission spectrum. As seen earlier, this method describes reasonably well both the LE and CT band shapes and allows determination of spectral properties with better accuracy. ${ }^{44-46,67-69}$

Shifts of the emission spectra from the peak of the reference emission spectra were estimated and added to the average peak frequency of the emission spectra to determine the emission peak frequencies of the LE and CT bands. The average of the reference emission peak frequency was calculated by averaging the numbers obtained by fitting the upper half of the reference emission spectrum with an inverted parabola, first moment and the arithmetic mean of the frequencies at half intensities on both blue and red ends of the emission spectrum. ${ }^{56,70}$ Absorption peak frequencies were obtained by calculating the first moments of the absorption spectra. The error associated with the peak frequency determination is typically $\pm 250 \mathrm{~cm}^{-1}$ and that with the band area is $\sim 10 \%$ (of the reported value), unless otherwise mentioned.

\subsection{Reaction time constant measurements}

Time correlated single photon counting (TCSPC) technique based on a laser system (Lifespec-ps, Edinburgh, UK) with a light emitting diode (LED) was used to collect fluorescence emission intensity decays. The excitation wavelength was $299 \mathrm{~nm}$ and the full width at half maximum (fwhm) of the instrument response function $($ IRF) $\sim 475$ ps. Emission decays were collected at both LE and CT peak positions (of steady state spectrum) with an emission band pass of $8 \mathrm{~nm}$ at magic angle of $54.7^{\circ}$ to avoid effects of solute rotation. ${ }^{71}$ Subsequently, the collected emission decays were deconvoluted from the IRF and fitted to bi-exponential functions of time using an iterative reconvolution algorithm. ${ }^{55}$ Such an iterative process is believed to be capable of describing dynamical events approximately 3-5 times faster than the fwhm of the IRF. ${ }^{48,72,73}$ For all $\mathrm{W}_{\mathrm{s}}$ values in these non-aqueous reverse micelles and all $\mathrm{LiClO}_{4}$ concentrations at $\mathrm{W}_{\mathrm{s}}=5$ for acetonitrile, bi-exponential fit to each of the LE emission decays produced one short time constant and one long time constant, whereas each of the CT emission decays (collected wherever possible) fit generated one rise-time (similar to short time constant of LE decay) and one long time constant. Therefore, the short time constant associated with the LE decay was considered as the reaction time. The LE decay of the TICT molecules in non-polar solvents such as in heptane or hexane was found to decay single exponentially with one time constant in the range of a couple of nanosecond. For a few cases, emission decays were collected at two or three different emission wavelengths around the LE and CT peaks and the analysed data were found to vary within a small uncertainty. All the experiments were performed at room temperature, $295 \pm 0.5 \mathrm{~K}$.

\subsection{Dynamic Stokes'shift measurements}

About 20 emission decays at equally spaced wavelengths across the steady-state emission spectrum of $\mathrm{C} 153$ (solute) dissolved in non-aqueous reverse micelles were collected at magic angle. The collected emission decays were first deconvoluted from the IRF to remove the instrumental broadening and then fitted with a multi-exponential function using an iterative reconvolution algorithm. Time resolved emission spectra (TRES) were then reconstructed from the decay fit parameters. ${ }^{55}$ The time dependent solvation of the laser excited probe was then followed by constructing the normalized spectral or solvation response function ${ }^{55}$

$$
S(t)=\frac{v(t)-v(\infty)}{v(0)-v(\infty)}
$$

where $v(x)$ denotes some measure (first moment or peak) of the time resolved emission spectrum at various time slices. While $v(0)$ represents the frequency of the dissolved solute's emission spectrum immediately after excitation, $v(\infty)$ denotes the frequency of the emission spectrum after the solvent relaxation is complete (the solute is still in its excited state). Solvation response function, $S(t)$ obtained experimentally was found to be multi-exponential functions of time and the average solvation times were obtained analytically as $\left\langle\tau_{s}\right\rangle=\int_{0}^{\infty} d t S(t)=\sum_{i=1}^{n} a_{i} \tau_{i}$.

\subsection{Rotation time measurements}

Emission decays for time-resolved fluorescence anisotropy $(r(t))$ were collected at the peak wavelength of the steady state emission bands. ${ }^{74}$ Time resolved fluorescence anisotropies, $r(t)$, were calculated from the collected and background subtracted parallel $\left(\mathrm{I}_{\mathrm{II}}(\mathrm{t})\right)$ and perpendicular $\left(\mathrm{I}_{\perp}(\mathrm{t})\right)$ decays by using the following well-known formula ${ }^{74}$

$$
r(t)=\frac{I_{I I}(t)-G I_{\perp}(t)}{I_{I I}(t)+2 G I_{\perp}},
$$

where $\mathrm{G}$ accounts for the differential sensitivity to the two polarizations which was obtained by tail matching the intensity decays $I_{I I}(t)$ and $I_{\perp}(t)$. Note that the 
average value for $\mathrm{G}$ obtained by tail matching the relevant decays at times longer than the anticipated rotation time is $1.15 \pm 0.05$.

The time resolved anisotropy spectra were constructed from the collected emission decays by using Eq. 2 and fitted to functions of the following general form

$$
r(\mathrm{t})=r(0)\left[\mathrm{a}_{1} \exp \left(-\mathrm{t} / \tau_{1}\right)+\left(1-\mathrm{a}_{1}\right) \exp \left(-\mathrm{t} / \tau_{2}\right)\right],
$$

where $\tau_{1}$ and $\tau_{2}$ in Eq. 3 represent the time constants associated with the decays of the components constituting $\mathrm{r}(\mathrm{t})$. Initial anisotropy was denoted by $\mathrm{r}(0)$ and was taken as 0.376 for fitting the time resolved anisotropies of $\mathrm{C} 153$ in all the concentration in non-aqueous reverse micelles studied here. ${ }^{74}$ Time integration of $r(t) / r(0)$ then produces the average rotation time: $\left\langle\tau_{r}\right\rangle=a_{1} \tau_{1}+$ $\left(1-a_{1}\right) \tau_{2}$.

\section{Results}

\subsection{Characterization of the reaction medium}

3.1a Estimation of the average $\varepsilon_{0}$ of the confined pool: As solution polarity strongly influences an ICT reaction mainly via modifying the reaction barrier, ${ }^{48}$ we have estimated the average dielectric constant $\left(\varepsilon_{0}\right)$ of the polar solvent confined in AOT/n-heptane/acetonitrile and AOT/n-heptane/methanol reverse micelles by using the following empirical relation ${ }^{10,56}$

$$
\begin{aligned}
v_{e m}\left(10^{3} \mathrm{~cm}^{-1}\right)= & v_{g}^{e m}-A\left[\left(\varepsilon_{0}-1\right) /\left(\varepsilon_{0}+2\right)\right] \\
& -B\left[\left(n^{2}-1\right) /\left(n^{2}+2\right)\right],
\end{aligned}
$$

where $v_{e m}$ represents the emission peak frequency of C153 dissolved in confined solvent pool, $\varepsilon_{0}$ the average static dielectric constant, $n$ the refractive index of the solvent. Fit ${ }^{10,56}$ to liquid data for $\mathrm{C} 153$ produces $v_{g}^{e m}=23.12, A=5.06$ and $B=1.5$. The value of $\varepsilon_{0}$ estimated by using Eq. 4 for non-aqueous solvent pools are shown as a function of $W_{s}$ in the upper panel of figure 1. It is evident form this panel that the estimated dielectric constants of confined acetonitrile and methanol at the largest $W_{s}$ are reduced by factors of $\sim 5$ and $\sim 3$, respectively. The reduction at smaller $W_{s}$ is even more drastic where the estimated dielectric constant is approximately an order of magnitude less than that at the bulk. Such a drastic reduction of bulk dielectric constant upon confinement has also been reported earlier for water ${ }^{10,75}$ and model solvents. ${ }^{76,77}$ This confinement-induced reduction of $\varepsilon_{0}$ can be under-
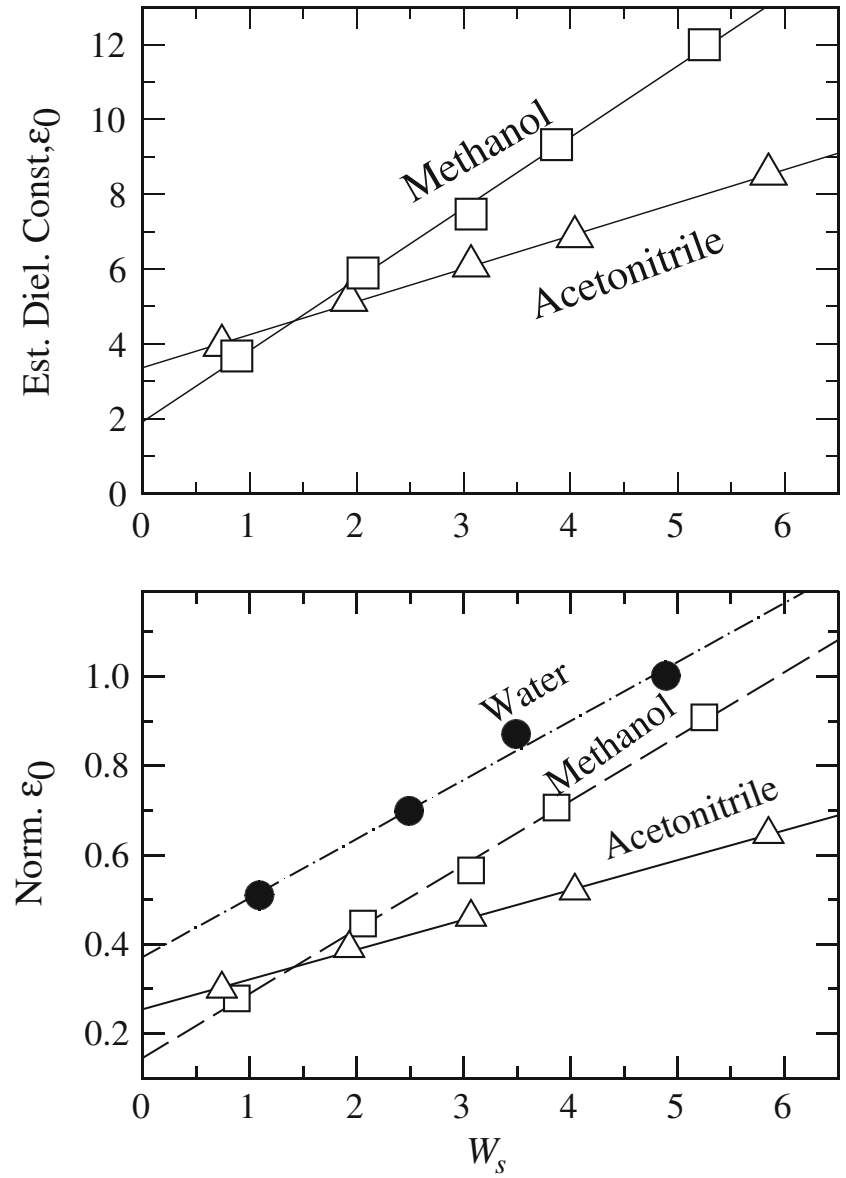

Figure 1. $W_{s}$ dependence of static dielectric constant $\left(\varepsilon_{0}\right)$ for confined solvents in AOT/n-heptane/acetonitrile and $\mathrm{AOT} / \mathrm{n}$-heptane/methanol reverse micelles in upper panel. Values of $\varepsilon_{0}$ at different $W_{s}$ values are obtained by using the fluorescence spectral shift of a non-reactive solvation probe, coumarin $153(\mathrm{C} 153)$ in Eq. 4 of the text. The lines going through the symbols (triangles and circles) are obtained from non-linear regression fits and can be described by the following parameters: for methanol, intercept $=1.92$, slope $=$ 1.90 and correlation coefficient $(\mathrm{R})=0.99$; for acetonitrile, intercept $=3.17$, slope $=0.93$ and correlation coefficient $(\mathrm{R})=0.99$. Comparison of the growth of $\varepsilon_{0}$ with $W_{s}$ among confined water, acetonitrile and methanol (lower panel). For each solvent, the estimated $\varepsilon_{0}$ has been normalized by the respective value at the highest pool-size shown here.

stood by recalling that the magnitude of the meansquared fluctuations in the collective dipole moment $\left(\left\langle\delta M^{2}\right\rangle=\left[\left\langle\mathbf{M}^{2}\right\rangle-<\mathbf{M}>^{2}\right]\right)$ determines $\varepsilon_{0}$ through the relation, ${ }^{78,79}\left[\varepsilon_{0}-1\right] \propto V^{-1}\left\langle\delta \mathbf{M}^{2}\right\rangle$, where the collective dipole moment of the system $(\mathbf{M})$ of volume $V$ is connected to the individual molecular dipole moment $\left(\mu_{i}\right)$ as follows, $\mathbf{M}=\sum_{i} \mu_{i}$. Since $\left\langle\delta M^{2}\right\rangle \sim N(N$ being the number of polar solvent molecules), it is only natural that the dielectric constant would be significantly reduced (compared to the bulk values) at smaller 
$W_{s}$ where lesser number of polar solvent molecules are present. This relation further suggests a linear increase of $\varepsilon_{0}$ of the confined solvent with $W_{s}$. This is indeed the case for methanol, acetonitrile and water as reflected in the data shown in the lower panel of figure 1. This linearity breaks down at higher values of $W_{s}$ for confined water ${ }^{10}$ probably because of solute's increasing expulsion from the core to the cavity interface due to interaction of the dipolar solute with ionic surface and increased hydrophobic interaction.

The observed linear dependence for confined acetonitrile on $W_{s}$ is interesting as earlier studies ${ }^{19}$ indicate confined solvent pool does not grow with $W_{s}$ when acetonitrile is used to form reverse micelles. However, several spectroscopic measurements ${ }^{19,34-38}$ using different coumarin dyes in these non-aqueous reverse micelles have reported red-shift in absorption and fluorescence spectra with $W_{s}$, shifts being relatively smaller for acetonitrile than those measured for methanol reverse micelles. Similar results have also been observed in the present study which are reflected in the linear increase of estimated $\varepsilon_{0}$ with $W_{s}$ and uniformly lower values of it (estimated $\varepsilon_{0}$ ) for confined acetonitrile than methanol between $1<W_{s}<6$. Moreover, absorption and fluorescence emission spectra recorded at $W_{s}$ values in this range for acetonitrile reverse micelles (figure S1, Supporting Information) do not indicate any solute partitioning between polar and non-polar phases. For methanol reverse micelles also, spectral shapes do not indicate such portioning (spectra not shown here). These observations are similar to earlier reports ${ }^{19,34-38}$ and, therefore, suggests that dipolar interactions among polar solvent molecules, solute-solvent dipolar interaction and interactions of the ionic surface with the dipolar solute and dipolar solvent molecules lead these species (dipolar solute and solvent) to reside largely inside the cavity.

The lower panel of figure 1 also indicates that the slopes of variation of dielectric constant with $W_{s}$ are similar for methanol and water but very different from that for acetonitrile. This may be linked to the partial restoration of hydrogen bonding (H-bonding) network in methanol at larger $W_{s}$, which is completely absent for the polar aprotic solvent, acetonitrile. The similar slopes for water and methanol further supports the notion that increase in $W_{s}$ induces progressive partial restoration of the confinement-disrupted H-bonding network, which, in turn, introduces steeper slopes for the increase of $\varepsilon_{0}$ in these network solvents. The confinement-induced disruption of $\mathrm{H}$-bonding network has been observed in many earlier studies and suggested to be responsible for depression of freezing transition temperature of water. ${ }^{80-84}$ It is to be mentioned here that the estimated dielectric constant reported here may not represent the 'true' value because ions present in the pool can also induce fluorescence shift, which, in the present calculation scheme, is considered as that by dipolar solvents. In addition, spectral shift depends on probe-location in such heterogeneous media and thus the estimated value may vary slightly if one changes the excitation wavelength. ${ }^{85,86}$

\section{1b Solvation time scale of confined polar solvent} pool: Average solvation times at several $W_{s}$ values for acetonitrile and methanol reverse micelles have already been measured via dynamic Stokes' shift experiments. ${ }^{34-38}$ Stokes' shift dynamics in confined methanol and acetonitrile have been found to be at least three orders of magnitude slower than that of the bulk. In addition, these studies report that dynamics become faster for methanol with $W_{s}$ but remains insensitive for acetonitrile. Our measurements using C153 as probe in AOT/n-heptane/acetonitrile reverse micelles at $W_{s}=5$ produce a dynamic Stokes' shift of $\sim 800 \mathrm{~cm}^{-1}$ which is in semi-quantitative agreement with the data reported earlier. ${ }^{34}$ The measured average solvation time $\left(<\tau_{s}>\right)$ at this pool size has been found to be $\sim 2000$ times slower compared to that for bulk acetonitrile with the same probe molecule. ${ }^{55}$ This is also in qualitative agreement with general results from dynamic Stokes' shift measurements for polar solvents under confinement. ${ }^{2-7,34-38}$

Although we have not repeated the measurements of $W_{s}$ dependent Stokes' shift dynamics for confined methanol and acetonitrile, we have measured the electrolyte concentration dependence of it in confined acetonitrile at $W_{s}=5$. The results are presented in figure 2 where the average solvation time $\left(<\tau_{s}>\right)$ shows a non-monotonic electrolyte $\left(\mathrm{LiClO}_{4}\right)$ concentration dependence. Note that the time scale obtained in our time-resolved measurements is significantly faster than what has been observed in similar pool size $\left(W_{s}=4\right)$ in the absence of added electrolyte. ${ }^{34}$ This is somewhat perplexing as addition of electrolyte is expected to slow down the rate of solvation. ${ }^{73}$ One reason could be that the present analyses consider spectral evolution only up to the time when $v(t)$ matches that from steady state fluorescence experiments $\left(v_{e m}\right)$. The consideration of $v(t=\infty) \equiv v_{e m}$ leaves out a dynamic Stokes' shift of $\sim 200 \mathrm{~cm}^{-1}$ from the total spectral relaxation. Moreover, this part has been found to relax with a time constant of a few to several nanoseconds. While this extremely slow relaxation occurring with a time 


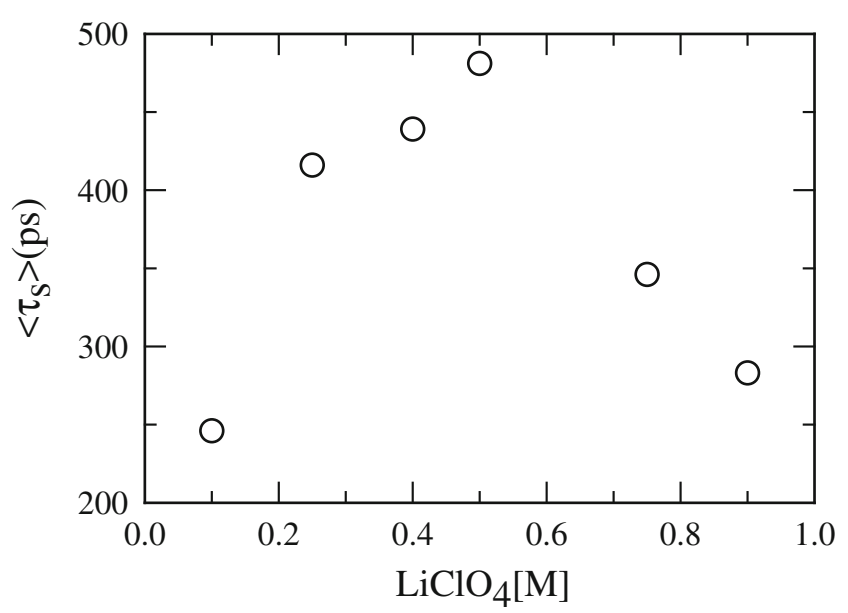

Figure 2. Electrolyte $\left(\mathrm{LiClO}_{4}\right)$ concentration dependence of average solvation time in AOT/n-heptane/acetonitrile reverse micelles at $W_{s}=5$. C153 has been used as a solvation probe.

constant of few to several nanoseconds may be real for these heterogeneous media, this small amount of dynamic shift $\left(\sim 200 \mathrm{~cm}^{-1}\right)$ can also arise from a modification in the vibronic structure of the line-shape due to difference in interaction with the environment of the probe in its ground and excited states. ${ }^{55}$

The increase in $\left\langle\tau_{s}>\right.$ at low $\mathrm{LiClO}_{4}$ concentration in figure 2 is because of enhancement of medium viscosity and increased participation of the diffusive ionsolvent exchange dynamics ${ }^{73}$ in the near vicinity of the dipolar probe. After a certain concentration, further addition of electrolyte may produce more number of ion-pairs which can significantly enhance the average polarity of the confined pool. Such a scenario has already been observed while studying Stokes shift dynamics in bulk ethyl acetate $\left(\varepsilon_{0} \sim 6\right)$ in presence of $\mathrm{LiClO}_{4}$ at various concentrations. ${ }^{42}$ Therefore, the decrease in $\left\langle\tau_{s}\right\rangle$ at higher electrolyte concentrations may be attributed to the increased average medium polarity and additional participation of rotational modes (via the rotation of ion-pairs). ${ }^{87}$ Note the decrease in $\left\langle\tau_{s}\right\rangle$ upon further addition of electrolyte at higher concentrations has also been observed in experiments with electrolyte solutions of strongly polar solvents. ${ }^{73}$ Application of Fee-Maroncelli method ${ }^{88}$ further suggests that the present experiments have missed, on an average, $\sim 40 \%$ of the total shift (table S1, Supporting Information) at this pool size for all these $\mathrm{LiClO}_{4}$ concentrations. It is to be mentioned here that dynamic light scattering (DLS) measurements indicate a fluctuation in the pool size with $\mathrm{LiClO}_{4}$ concentration but the size fluctuation always remains within $\pm 20 \%$ of the pool diameter in the absence of electrolyte.

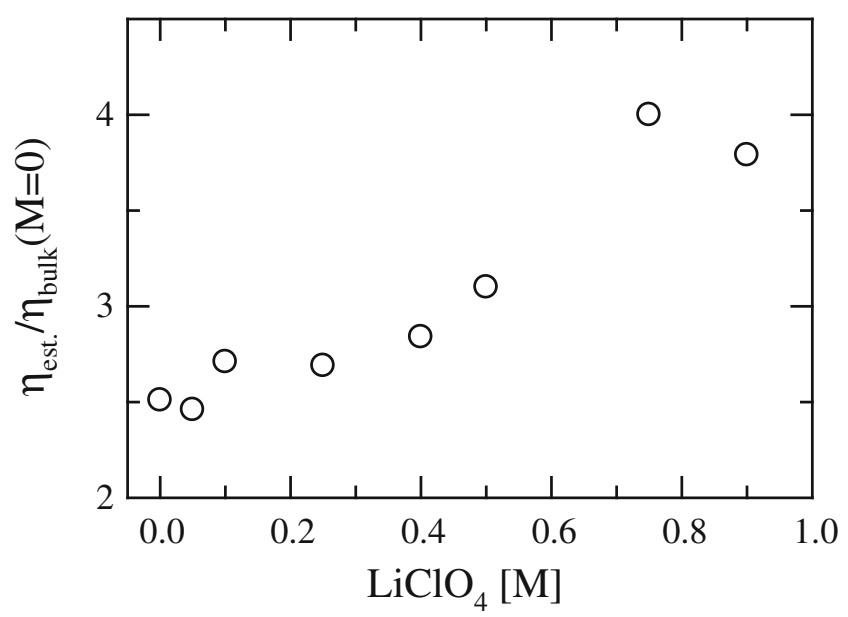

Figure 3. Electrolyte-induced enhancement of pool viscosity in AOT/n-heptane/acetonitrile reverse micelles at $W_{s}=5$. Ratio between the estimated viscosity $\left(\eta_{\text {est }}\right.$. $)$ of confined acetonitrile at this $W_{s}$ and that $\left(\eta_{b u l k}[M=0]\right)$ of bulk pure acetonitrile are shown as a function of electrolyte $\left(\mathrm{LiClO}_{4}\right)$ concentration. Values of $\eta_{\text {est }}$. have been determined from the measured rotation times of $\mathrm{C} 153$ dissolved in the reverse micelle droplets.

3.1c Estimation of local viscosity: Local viscosity experienced by the probe in reverse micelles droplets has been estimated by using the correlation, $\left\langle\tau_{r}\right\rangle=$ $(58.1 \pm 1.6) \eta^{0.96 \pm 0.03}$, constructed from the rotation data of the same probe in bulk polar protic and aprotic solvents at room temperature. ${ }^{74}$ Note in the above correlation, average rotational time $\left\langle\tau_{r}\right\rangle$ is in picosecond

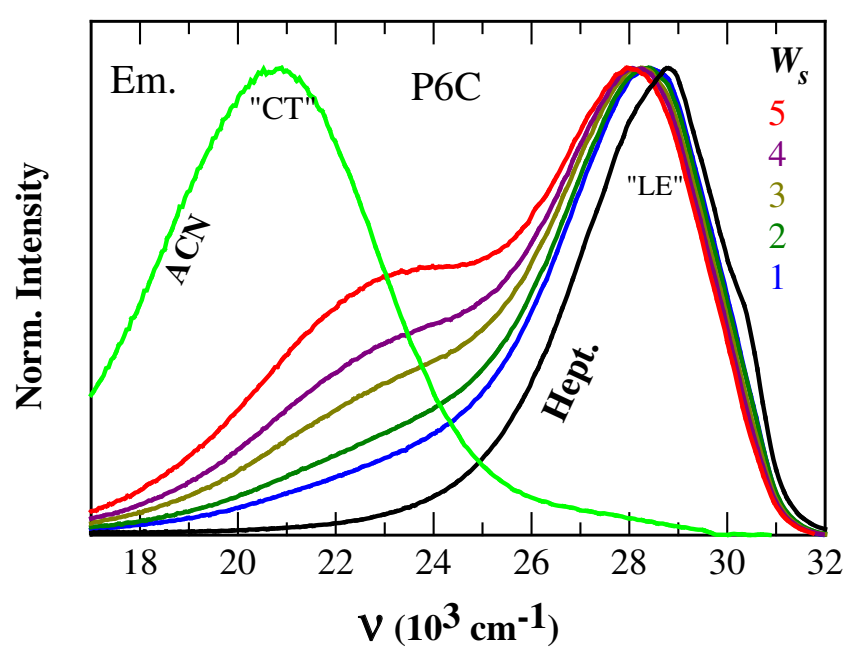

Figure 4. $W_{s}$ dependence of fluorescence emission spectrum of one of the ICT molecules, P6C dissolved in acetonitrile reverse micelles. The spectra are colour-coded. Emission spectra of the same molecule in acetonitrile and heptane are also shown. 'LE' represents the locally excited state and 'CT' the charge-transferred state. 
(ps) and $\eta$ is in centipoise $(\mathrm{cP}) .{ }^{89}$ Our measured $\left\langle\tau_{r}\right\rangle$ for $\mathrm{C} 153$ in AOT/heptane/acetonitrile reverse micelles at $W_{s}=5$ in the absence of any added electrolyte compares well with the existing data $(\sim 60$ ps versus $\sim 80 \mathrm{ps}$ at $W_{s}=4^{35}$ ). Corresponding bi-exponential fit parameters for the measured $r(t)$ are summarized in table S2 (Supporting Information). Electrolyte concentration dependent viscosity of the confined acetonitrile at $W_{s}=5$ is then extracted by inserting the measured $\left\langle\tau_{r}\right\rangle$ into the above correlation. The results are presented in figure 3 where a near linear increase of estimated viscosity with $\mathrm{LiClO}_{4}$ concentration is indicated. Note the estimated viscosity $\left(\eta_{\text {est }}\right)$ at $W_{s}=5$ in the absence of electrolyte is nearly twice as large as that of bulk pure acetonitrile $\left(\eta_{\text {bulk }}[M=0]=0.34 \mathrm{cP}\right)$ which, upon further addition of electrolyte, enhances by a factor of 4 . However, such an interpretation of slowed-down rotation time of a polar probe in terms of enhanced viscosity assumes negligible or no frictional resistance arising from the longer-ranged solutesolvent dipolar interaction and solute-ion dipole-ion interaction. $^{90}$ A number of experimental ${ }^{74,91}$ and simulation studies ${ }^{92-94}$ in highly polar conventional solvents have shown that longer-ranged interactions play a minor role in rotational friction. Mechanical friction from short-ranged interactions, its enhancement via electrostatic interactions (or electrostriction), and in some cases, specific solute-solvent interactions, principally govern the rotational time scale.

\subsection{ICT reaction in confined polar solvent}

3.2a Reaction equilibrium constant and free energy ( $W_{s}$ dependence): Representative emission spectra of $\mathrm{P} 6 \mathrm{C}$ in $\mathrm{AOT} / \mathrm{n}$-heptane/acetonitrile reverse micelles at different $W_{s}$ values are shown in figure 4 . For comparison, emission spectra of the same ICT molecule in acetonitrile and heptane are also shown in the same figure. It is evident from this figure that as $W_{s}$ increases, the emission spectrum red-shifts with simultaneous growth in the charge-transferred (CT) band. The CT band

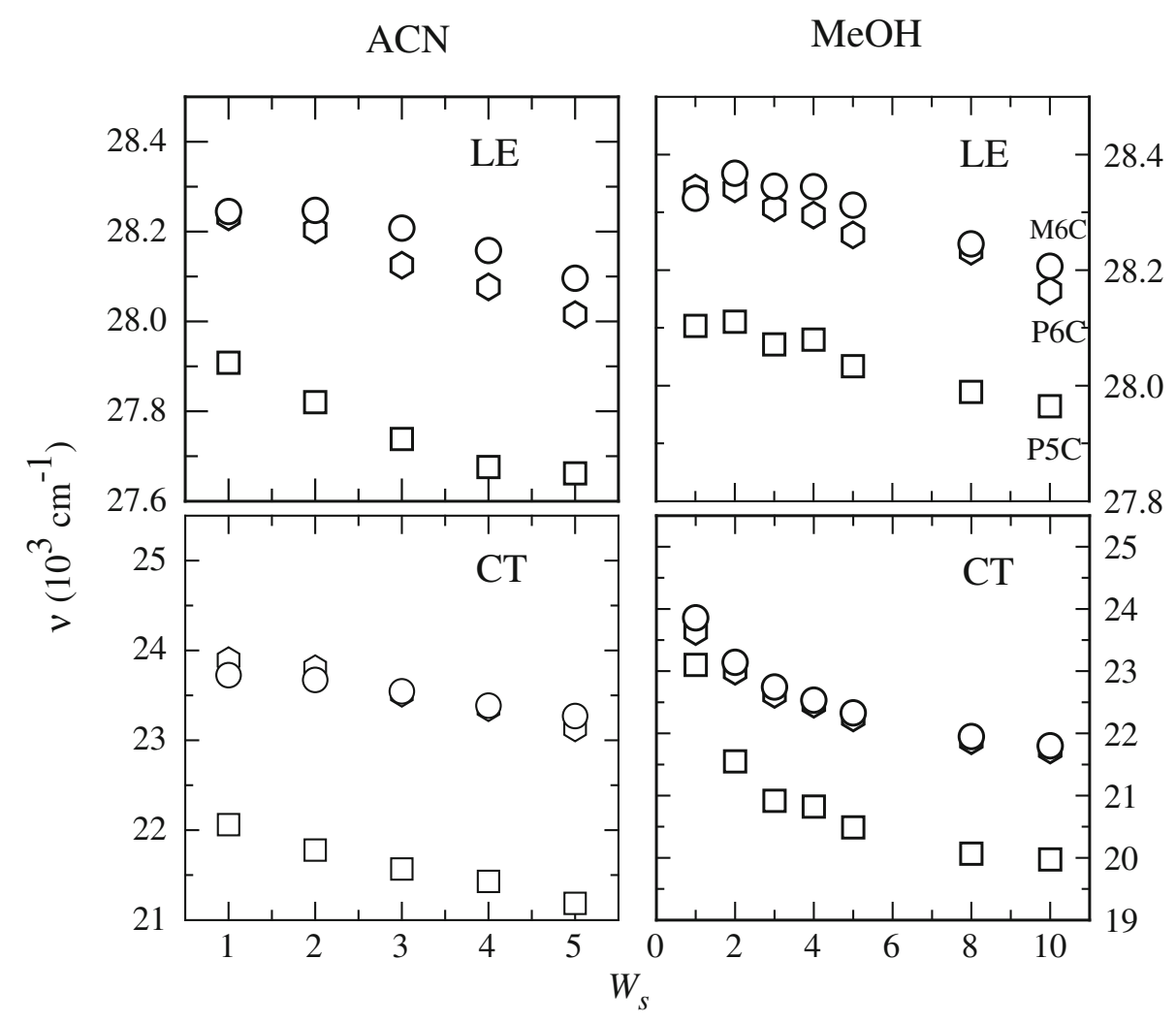

Figure 5. $W_{s}$ dependence of the LE and CT emission peak frequencies of P5C, P6C and $\mathrm{M} 6 \mathrm{C}$ in AOT/n-heptane/acetonitrile and AOT/n-heptane/methanol. Non-aqueous solvents acetonitrile (left panel, 'ACN') and methanol (right panel, 'MeOH') are indicated. Symbols (circles, hexagons and squares) tagged with solute identity in the upper-right panel describe the data for P5C, P6C and M6C in all the four panels. The estimated uncertainty in frequency is $\pm 300 \mathrm{~cm}^{-1}$. 
becomes most prominent at the highest $W_{s}$ considered. Since it is known that medium polarity facilitates the formation of CT state, ${ }^{10}$ the continuous growth in CT population with $W_{s}$ may be attributed to the continuous enhancement of medium polarity at higher loading of polar solvent. The role of medium polarity in forming the CT population is further supported by the observation that photo-excitation of M6C predominantly produces either $\mathrm{CT}$ population in bulk acetonitrile $\left(\varepsilon_{0} \sim 36\right)$ or LE in bulk heptane $\left(\varepsilon_{0} \sim 2\right)$. Spectral shift and $\mathrm{CT}$ band formation, therefore, indicate that the ICT molecule (P6C in this case) is indeed trapped in the solvent pool and interacting with the immediate environment. However, solute partitioning into the non-polar and micellar pseudophases ${ }^{9,29}$ could be an issue here as this will lead to emission at different energies producing an emission profile similar to the ones displayed in figure 4. However, the peak-to-peak difference between the emission spectra of $\mathrm{P} 6 \mathrm{C}$ in acetonitrile and heptane is much larger $\left(\sim 8000 \mathrm{~cm}^{-1}\right)$ than that in the largest pool ( $\sim 5000 \mathrm{~cm}^{-1}$, see also figure 5$)$. In addition, the peak frequency of the CT emission band at any of the $W_{s}$ considered is blue-shifted at least by $\sim 3000 \mathrm{~cm}^{-1}$ than that in bulk acetonitrile. Moreover, the absorption spectra of this molecule at various $W_{s}$, shown in figure S2 (Supporting Information) do not indicate any distribution of the polar solvent out into the non-polar phase. All these observations suggest that the emission spectra of P6C in these reverse micelles are predominantly originating from those dissolved inside the confined polar solvent pool.

The $W_{s}$ dependences of the LE and CT emission peak frequency of the three TICT molecules in AOT/ n-heptane/acetonitrile and AOT/n-heptane/methanol reverse micelles are shown in figure 5 . In both these non-aqueous reverse micelles, the LE and CT peak

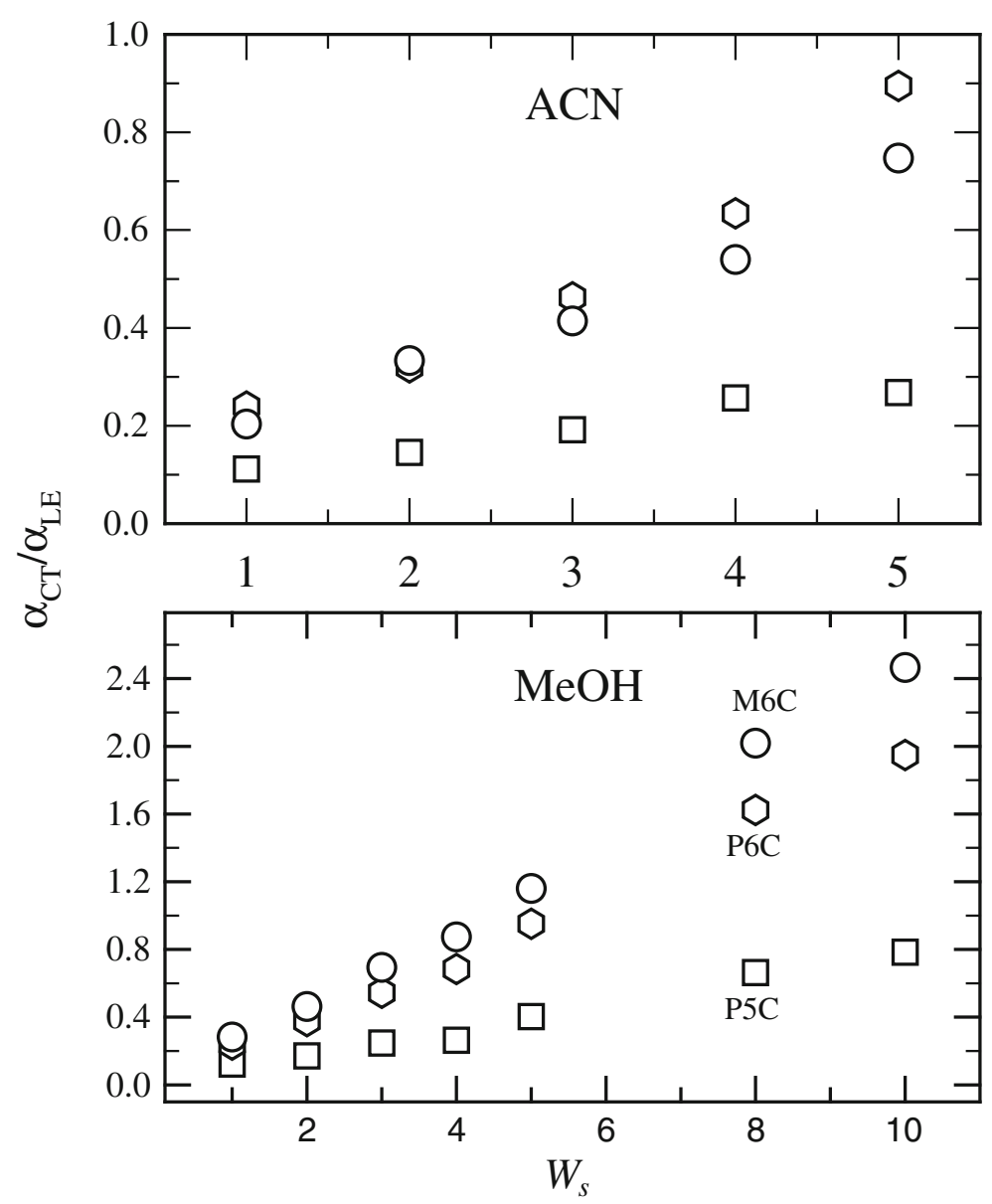

Figure 6. $W_{s}$ dependence of the ratio between the areas under the CT and LE emission bands $\left(\alpha_{C T} / \alpha_{L E}\right)$ of P5C, P6C and M6C in AOT/ n-heptane/acetonitrile and AOT/n-heptane/methanol. Identity of the non-aqueous polar solvents used is indicated in each panel. The uncertainty for CT band area is typically within $\pm 10 \%$. 
frequencies show red-shift with increase in pool size (see also table S3, Supporting Information). The total shift for the LE emission peak is much less and it is about $\sim 200 \mathrm{~cm}^{-1}$ for all these ICT molecules in these two types of reverse micelles. The total CT shift, on the other hand, is much larger and is $\sim 1000 \mathrm{~cm}^{-1}$ for both acetonitrile and methanol at $W_{s}=5$. In both these non-aqueous reverse micelles the regularity in spectral shifts (particularly for the CT band) with $W_{s}$ again suggests continuous increase of polarity around the trapped solute. The CT shift becomes even larger $(\sim 2000$ $3000 \mathrm{~cm}^{-1}$ ) for methanol because one can reach $W_{s}=$ 10 with this solvent. Interestingly, the total CT shift in methanol reverse micelles is very similar to earlier observations for M6C in aqueous reverse micelles. ${ }^{10}$ However, the LE peak frequency of closely related ICT molecules in neat solvents ${ }^{48}$ exhibited blue-shift with concomitant narrowing of the emission band, whereas in these non-aqueous reverse micelles the LE band redshifts with simultaneous broadening (see table S3). While the heterogeneity in the environment around the confined reactant in these reverse micelles can contribute to the spectral broadening, the observed redshift in LE band may arise due to the spectral broadening rather than from any fundamental difference in soluteenvironment interaction between neat and confined solvents. However, the uniform nature of the LE peak frequency versus LE-width correlation observed in the

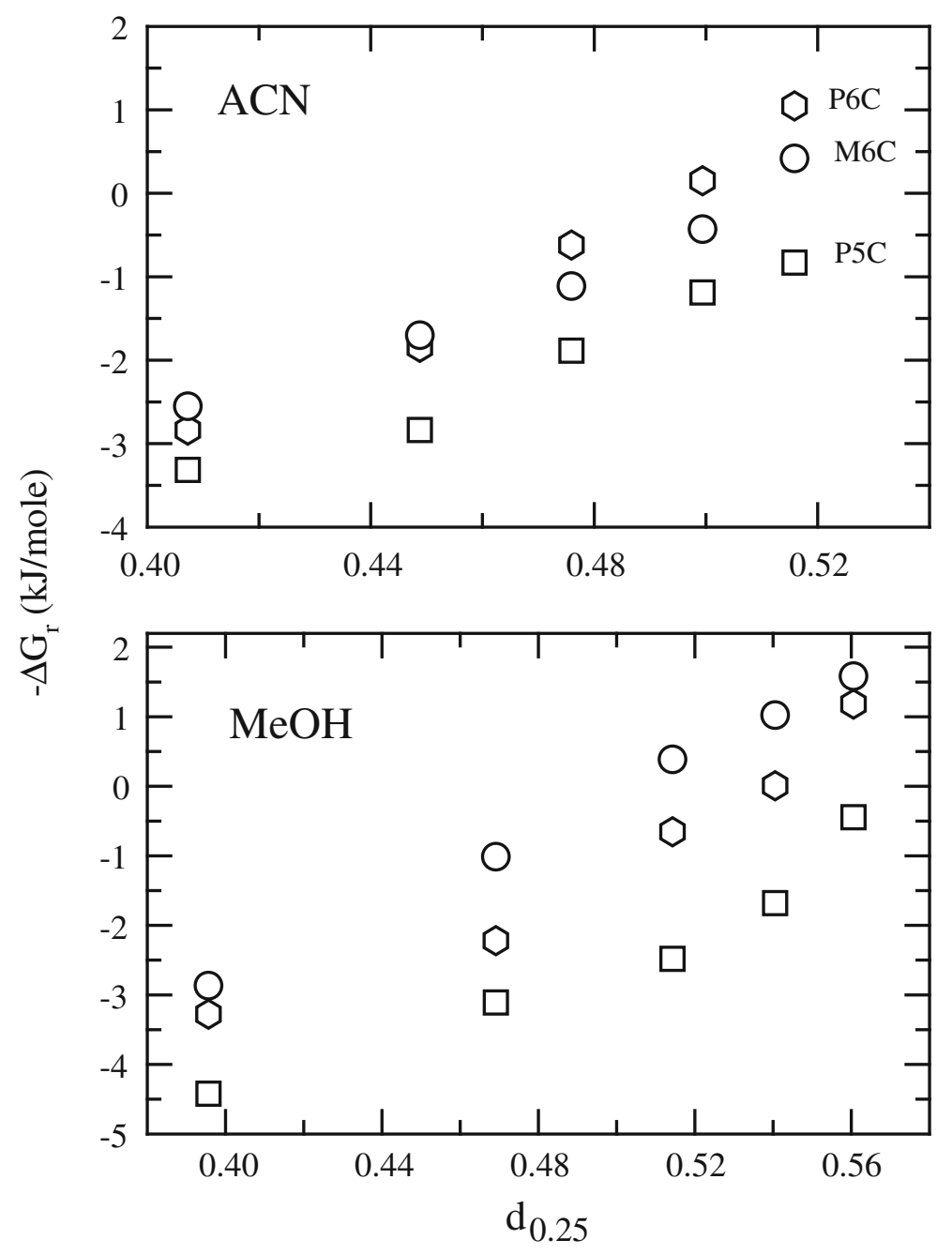

Figure 7. The correlation between the reaction driving force $\left(-\Delta G_{r}\right)$ and a dielectric field factor, $\mathrm{d}_{0.25}$ for the $\mathrm{LE} \rightarrow \mathrm{CT}$ conversion reaction of P5C, P6C and M6C molecules in AOT/n-heptane/acetonitrile (upper panel, 'ACN') and AOT/n-heptane/methanol (lower panel, 'MeOH') reverse micelles. Values of the dielectric constant for the confined solvent used are those shown in figure 1 . The symbols (hexagon, circle and square) are solute-tagged. 
present study warrants further investigation. The correlation between CT peak shift and bandwidth for these molecules in these reverse micelles is similar as that observed not only in aqueous reverse micelles ${ }^{10}$ but in bulk polar solvents ${ }^{48,68,69}$ and electrolyte solutions ${ }^{45,46,67}$ also.

Since the photo-induced $\mathrm{LE} \rightarrow \mathrm{CT}$ reaction in these molecules is favoured by the polarity of a reaction medium, ${ }^{48}$ the ratio between the areas under these two bands $\left(\alpha_{C T} / \alpha_{L E}\right)$ is expected to increase with $W_{s}$. Figure 6 reflects indeed such results where $\alpha_{C T} / \alpha_{L E}$ for P5C, P6C and M6C in these non-aqueous reverse micelles are shown as a function of $W_{s}$. The area ratios for these ICT molecules have been drastically reduced under confinement compared to those in the respective bulk polar solvents. For example, $\alpha_{C T} / \alpha_{L E}$ for P6C in the smallest and largest $W_{s}$ values for acetonitrile correspond to those found in bulk diethyl ether $\left(\varepsilon_{0} \sim 4\right)$ and bulk decanol $\left(\varepsilon_{0} \sim 7\right)^{48}$ but $\sim 100$ to $\sim 10$ times less than that in bulk acetonitrile. Moreover, in the acetonitrile-saturated solution of heptane $\alpha_{C T} / \alpha_{L E}$ for $\mathrm{P} 6 \mathrm{C}$ is found to be $\sim 4$ times less than that at $W_{s}=5$. Data shown in the lower panel of figure 6 indicate substantial reduction in $\alpha_{C T} / \alpha_{L E}$ for these ICT molecules in confined methanol also. The reduction in $\alpha_{C T} / \alpha_{L E}$ values in these confined solvents therefore follow the trend of change in $\varepsilon_{0}$ upon confining these polar solvents but the extent of decrease is much larger than the corresponding decrease in $\varepsilon_{0}$. This indicates that the solvent control of an ICT reaction in confined solvents is more complicated than that when the reaction occurs in bulk media.

Next, a correlation between the change in free energy for the photo-induced $\mathrm{LE} \rightarrow \mathrm{CT}$ conversion reaction $\left(-\Delta G_{r}\right)$ in these ICT molecules and a dielectric reaction field factor $\left(d_{c}\right)$ is explored in figure 7 for these non-aqueous reverse micelles. The following representative field factor, $d_{c}\left(\varepsilon_{0}\right)=\frac{\varepsilon_{0}-1}{2(1-c) \varepsilon_{0}+(1+2 c)}$, is employed for such a correlation where the $W_{s}$ dependent $\varepsilon_{0}$ is used. The parameter $c$, ranging between 0 and 1 , effectively accounts for solute polarizability effects. ${ }^{48} \mathrm{~A}$ value of 0.25 for $c$ has been used here since this has been found to produce the best correlation for reactions in bulk solvents. ${ }^{48}$ The driving force $\left(-\Delta G_{r}\right)$ for the reaction is obtained from the reaction equilibrium constant $\left(K_{e q}\right)$ by using the relation, where $K_{e q}$ has been calculated from the arithmetic mean of the equilibrium constant determined from the steady state data ${ }^{48}\left(K_{e q}^{\phi} \approx \frac{\alpha_{C T} v_{L E}^{3}}{\alpha_{L E} v_{C T}^{3}}\right)$ and time-resolved experiments $\left(K_{e q}^{a}=a_{r x n} / a_{d e c}\right)$. Note that $a_{r x n}$ and $a_{d e c}$ are respectively the amplitudes associated with the short (reaction) time and the long time components of the time-resolved emission (population) decay. Note that the $W_{s}$ dependent $-\Delta G_{r}$ varies from negative to positive indicating that a less favourable $\mathrm{LE} \rightarrow \mathrm{CT}$ conversion reaction in smaller $W_{s}$ becomes more favourable in larger $W_{s}$. In addition, a near linear correlation between $-\Delta G_{r}$ and $d_{c}$ suggests that the trapped reactants are experiencing more polar environment upon increasing $W_{s}$, and medium polarity influences an ICT reaction in confined solvents in qualitatively the same way as in bulk media.

\section{$3.2 \mathrm{~b}$ Effects of salt concentration on reaction at a} fixed $W_{s}$ : Effects of electrolyte on $\mathrm{LE} \rightarrow \mathrm{CT}$ conversion reaction occurring in confined polar solvent pool is studied next. Figure 8 depicts the $\mathrm{LiClO}_{4}$ concentration dependences of area ratio $\left(\alpha_{C T} / \alpha_{L E}\right)$ and reaction driving force $\left(-\Delta G_{r}\right)$ for the conversion reaction in all these molecules in AOT/heptane/acetonitrile reverse

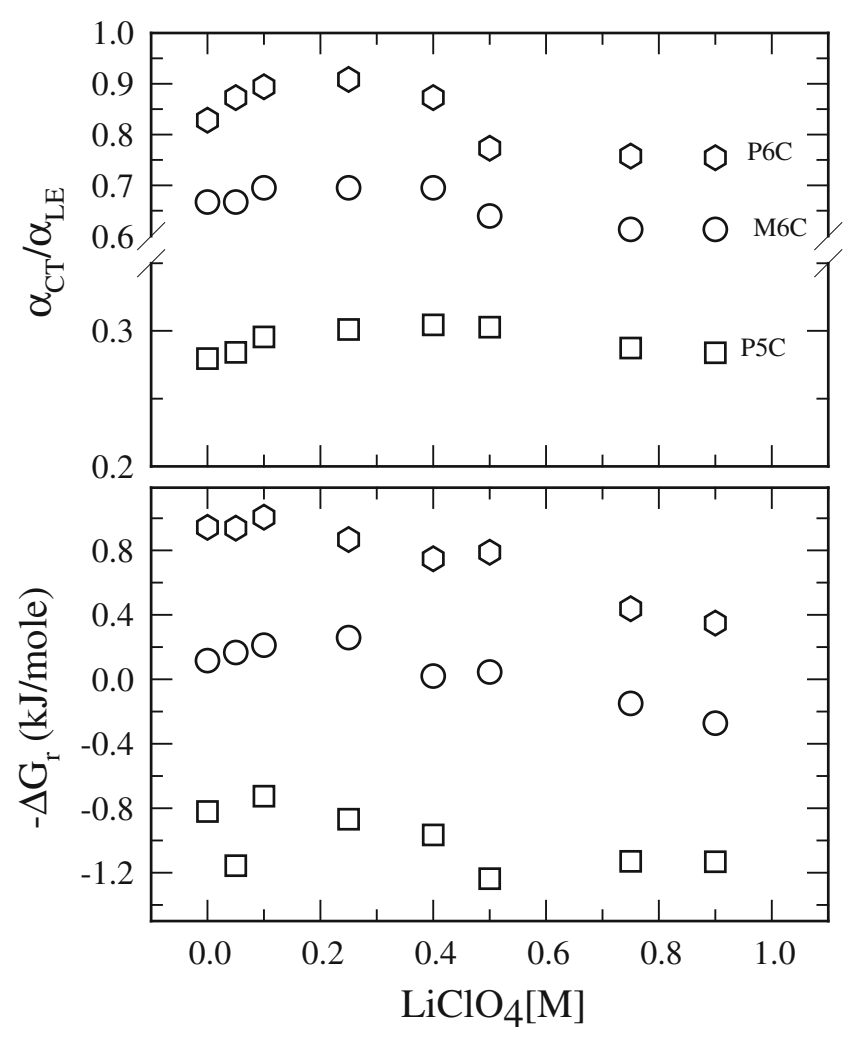

Figure 8. Electrolyte $\left(\mathrm{LiClO}_{4}\right)$ concentration dependence of area ratio, $\alpha_{C T} / \alpha_{L E}$ and reaction driving force $\left(-\Delta G_{r}\right)$ in these ICT molecules in AOT/heptane/acetonitrile reverse micelles at $W_{s}=5 .-\Delta G_{r}$ is obtained from the average equilibrium constants which is an arithmetic mean of the equilibrium constants determined from the steady state and time resolved experiments. As before, symbols are solute-tagged. 
micelles at $W_{s}=5$. Electrolyte effects on peak frequencies and widths are found to be very small (see figure S3, Supporting Information). However, it is evident from the upper panel of figure 8 that the area ratio varies non-monotonically with electrolyte concentration, degree of non-monotonicity being the strongest for P6C and weakest for P5C. A small variation in activation energies ${ }^{48}$ of these molecules and the subsequent difference in medium control might be responsible for such a behaviour. The skewedness is somewhat softened in the lower panel because of logarithmic relationship between $-\Delta G_{r}$ and $\alpha_{C T} / \alpha_{L E}$. Interestingly, such a non-monotonic dependence of average solvation time on electrolyte concentration at this pool size has already been observed (figure 2) and it is quite likely that these two are connected. We shall explore more on this issue when we describe results from time-resolved studies.

3.2c Reaction rate constant: $W_{s}$ dependence: The $W_{s}$ dependence of the $\mathrm{LE} \rightarrow \mathrm{CT}$ reaction rate constant of P5C, P6C and M6C has been studied in both AOT/hetpane/acetonitrile and AOT/heptane/methanol reverse micelles. Time-resolved fluorescence intensity decays for M6C in methanol reverse micelles at a few $W_{s}$ values are shown in figure 9. The decay pattern reflects a considerable $W_{s}$ dependence. Similar $W_{s}$ dependence of intensity decay has also been observed for reaction occurring in confined acetonitrile (decays not shown here). As already discussed, the reaction rate constants have been determined by fitting the collected LE and CT emission intensity decays to bi-exponential functions of time. A pair of such representative fits are displayed in figure 10 for $\mathrm{M} 6 \mathrm{C}$ in methanol reverse micelles at $W_{s}=5$. The fitted data along with the instrument response function and the residuals are also shown in the same figure. Similar values for the fast time constant $\left(\tau_{1}\right)$ associated with the LE-decay and CT-rise suggest that $\tau_{1}$ indeed represents the reaction time constant $\left(\tau_{r x n}=1 / k_{r x n}, k_{r x n}\right.$ being the reaction rate constant) associated with the $\mathrm{LE} \rightarrow \mathrm{CT}$ conversion reaction. In addition, adequacy of just two exponentials in describing the decay kinetics also suggests that the reaction proceeds via the same mechanism in these reverse micelles as observed in bulk acetonitrile and methanol. ${ }^{48,49}$ Most interestingly, the reaction time constant of $\sim 450 \mathrm{ps}$ at this $W_{s}$ is $\sim 30$ times longer than that measured with the same molecule (M6C) in bulk methanol $(\sim 15$ ps $) .{ }^{49}$ In saturated methanol or acetonitrile solutions of heptane, $\tau_{r x n}$ for M6C has been found to be $\sim 10$ times longer than in bulk pure polar solvents. This lengthening of $\tau_{r x n}$ in saturated binary mixtures is mainly due to the reduction in solution polarity. ${ }^{52-54}$ The fact that $\tau_{r x n}$ at $W_{s}=5$ is $\sim 3$ times larger than that in binary mixtures $(\sim 450$ ps versus

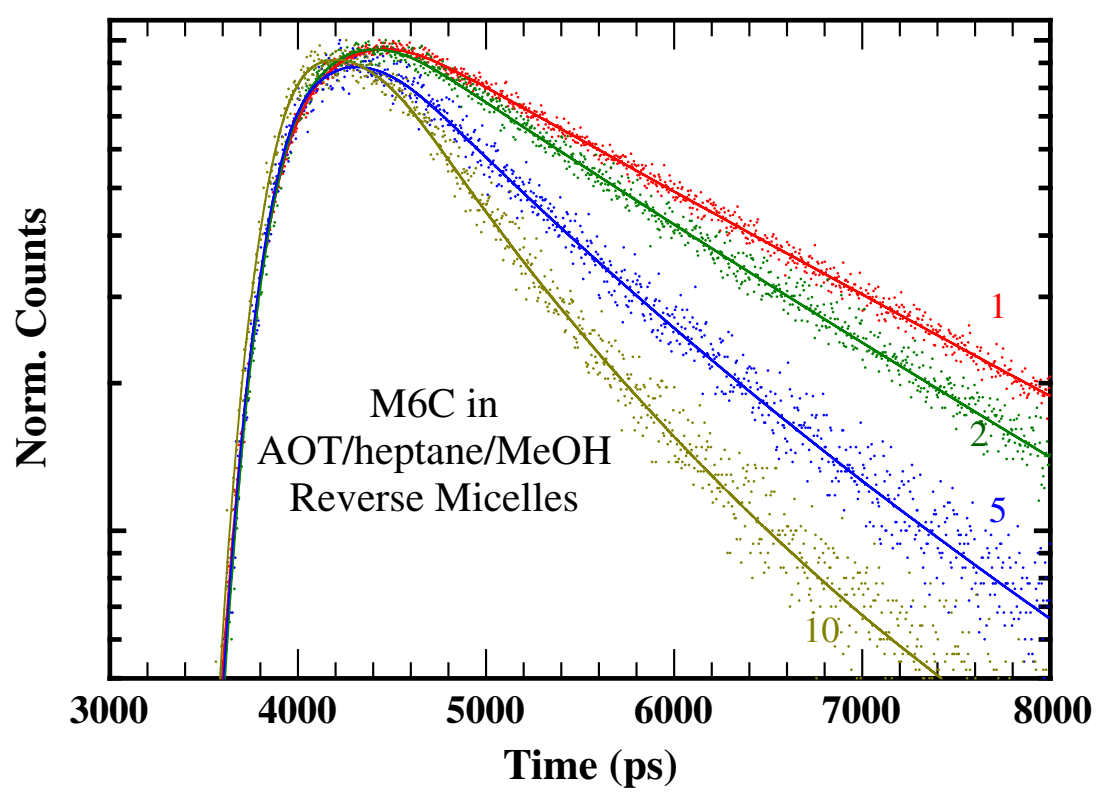

Figure 9. Pool-size dependence of time-resolved population decay of M6C in AOT/heptane/methanol reverse micelles. The figure is colour-coded. Number attached to each decay denotes a value of $W_{s}$ at which intensity decay has been collected. 

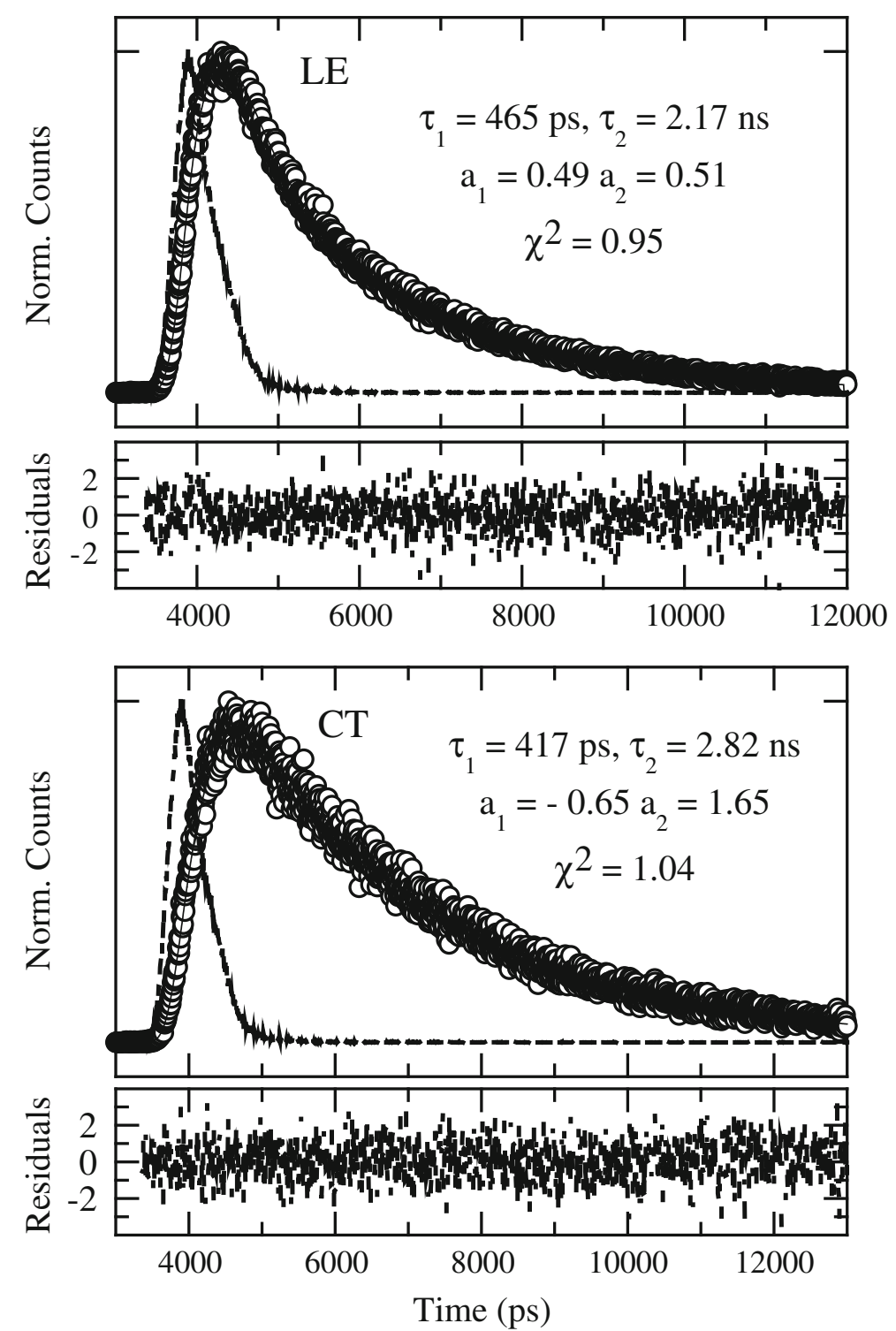

Figure 10. Representative LE emission decay (upper panel) and CT emission decay (lower panel) of M6C in AOT/n-heptane/methanol reverse micelles at $W_{s}=5$ and their bi-exponential fits. While the experimental data are represented by circles, fits through the data points are shown by solid lines. The instrument response function is shown by the broken line. $\chi^{2}$ denotes the 'goodness-of-fit' parameter. The LE peak count is $\sim 3000$ and CT peak count is $\sim 1000$. Residuals are shown in the bottom of each panel.

$\sim 150 \mathrm{ps}$ ) clearly indicates the measured reaction time is indeed associated with the reaction occurring in confined polar solvents.

While the $W_{s}$ dependent reaction time constants $\left(\tau_{r x n}\right)$ are given in Supporting Information (figure S4), the ratio between the reaction time constants in confined and bulk solvents, $\tau_{r x n}(R M) / \tau_{r x n}$ (bulk), obtained for various $W_{s}$ values are shown in figure 11. Data shown in this figure indicate substantial lengthening of $\tau_{r x n}$ in these non-aqueous reverse micelles for all the ICT molecules considered here and the extent of lengthening, depending on solute and $W_{s}$, ranges between $\sim 100$ and $\sim 10$. In addition, $\tau_{r x n}$ becomes faster by nearly a factor of 2 in these reverse micelles for changing $W_{s}$ from 1 to 5. Similar changes in 'local viscosity' are also reflected in the pool-size dependent rotation times measured with polar probes in these non-aqueous reverse micelles. ${ }^{34,38,95}$ For methanol, this enhancement 


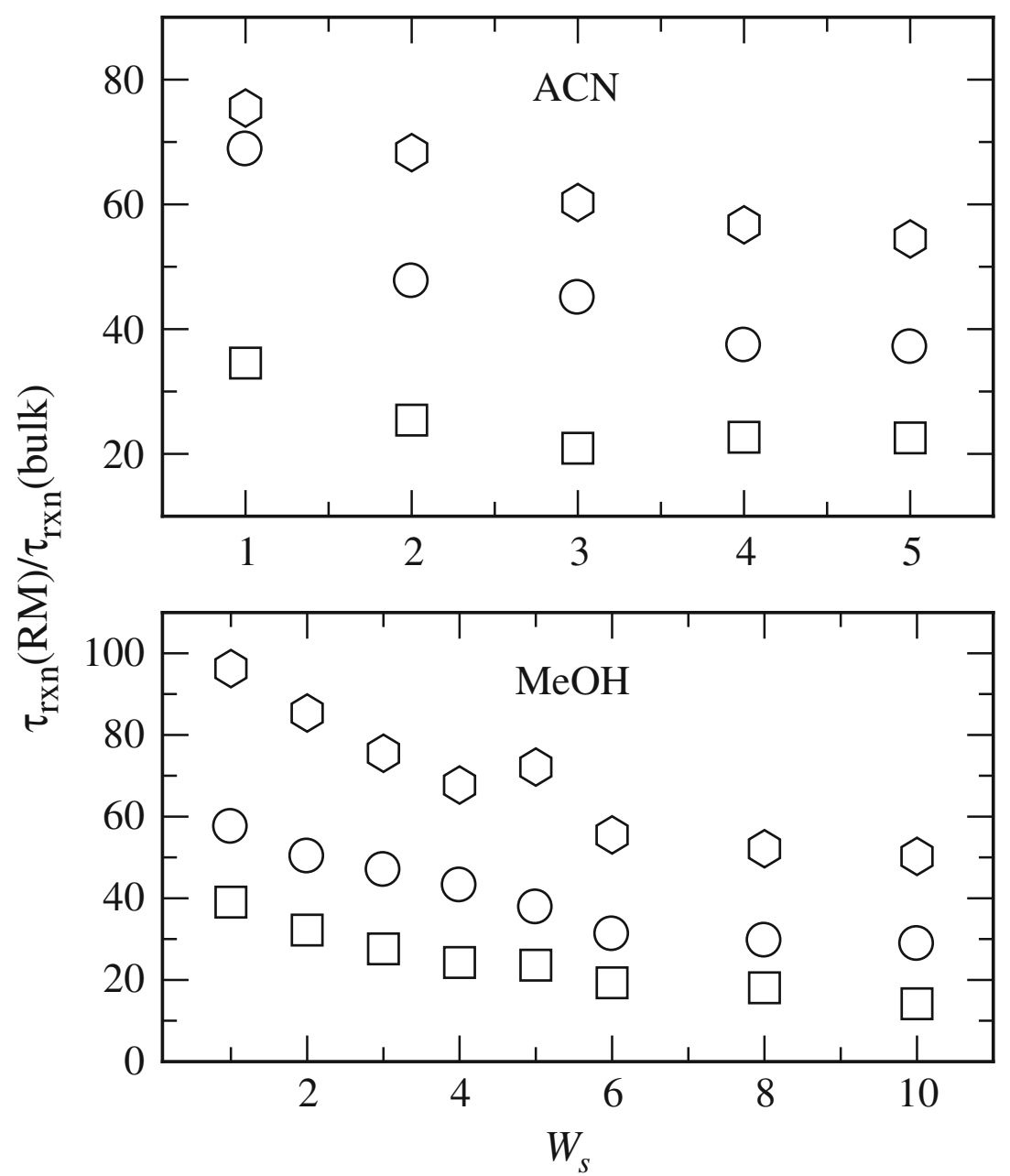

Figure 11. $W_{s}$ dependence of ratio between reaction time constant, $\tau_{r x n}(R M) / \tau_{r x n}($ bulk), associated with the $\mathrm{LE} \rightarrow \mathrm{CT}$ conversion reaction of P5C, P6C and M6C in AOT/n-heptane/acetonitrile (upper panel) and AOT/nheptane/methanol reverse micelles (lower panel), and reaction time in bulk acetonitrile and methanol. Note that the squares, hexagons, circles represent data respectively for $\mathrm{P} 5 \mathrm{C}, \mathrm{P} 6 \mathrm{C}$ and $\mathrm{M} 6 \mathrm{C}$. Error associated with the reaction time constants is typically $\pm 5 \%$ of the reported values.

factor doubles to $\sim 4$ as one can go up to $W_{s}=10$ with this alcohol solvent. Interestingly, earlier measurements ${ }^{37}$ of the non-radiative rate constant for $\mathrm{C} 152$ in methanol and acetonitrile reverse micelles showed much less reduction and very weak dependence on $W_{s}$. Therefore, the observed huge lengthening of $\tau_{r x n}$ upon confinement and substantial variation of it $\left(\tau_{r \times n}\right)$ with $W_{s}$ are new results and completely different from earlier reports. ${ }^{37}$ The presence of a sizeable activation barrier and tuning of it with solution polarity, and coupling of the twisting (reactive) mode to local viscosity have probably made the $\mathrm{LE} \rightarrow \mathrm{CT}$ conversion reaction of these ICT molecules more sensitive to both the confinement and size of it.
Although $\tau_{r x n}$ exhibits acceleration by a factor 2 to 4 upon increasing $W_{s}$ and follows the associated viscosity changes, the huge reduction of it over the bulk values suggests a much stronger dynamical solvent control of the $\mathrm{LE} \rightarrow \mathrm{CT}$ reaction in these ICT molecules. This can be understood qualitatively from the following example. At $W_{s}=5$, the solvent polarity (sensed by the solute) is reduced by a factor of $\sim 5$ and the viscosity enhanced by a factor of $\sim 2$. Had these two factors been the only reasons to influence the ICT reaction in M6C and acted in multiplicative manner, then the slowing down of $\tau_{r x n}$ (over the bulk value) at $W_{s}=5$ would have been merely a factor of $\sim 10$. The extent of reduction being much larger (ranging between 100 and 
20) than this only supports the conjecture that severely slowed down solvation response (by a factor of 1000 or even more) in these confined systems does not allow the reaction to occur in a fully solvent-relaxed state. This, in turn, indicates a critical role for slow solvation response in determining the rate of ICT reaction occurring in confined media. Further study is thus required to quantify and separate out the effects of slow solvation on such reactions occurring in reverse micelles.

3.2d Reaction at a fixed $W_{s}$ : Electrolyte effects: Figure 12 shows the electrolyte $\left(\mathrm{LiClO}_{4}\right)$ concentration dependence of reaction time constant $\left(\tau_{r x n}\right)$ for P5C, P6C and $\mathrm{M} 6 \mathrm{C}$ in $\mathrm{AOT} / \mathrm{n}$-heptane/acetonitrile reverse micelles at

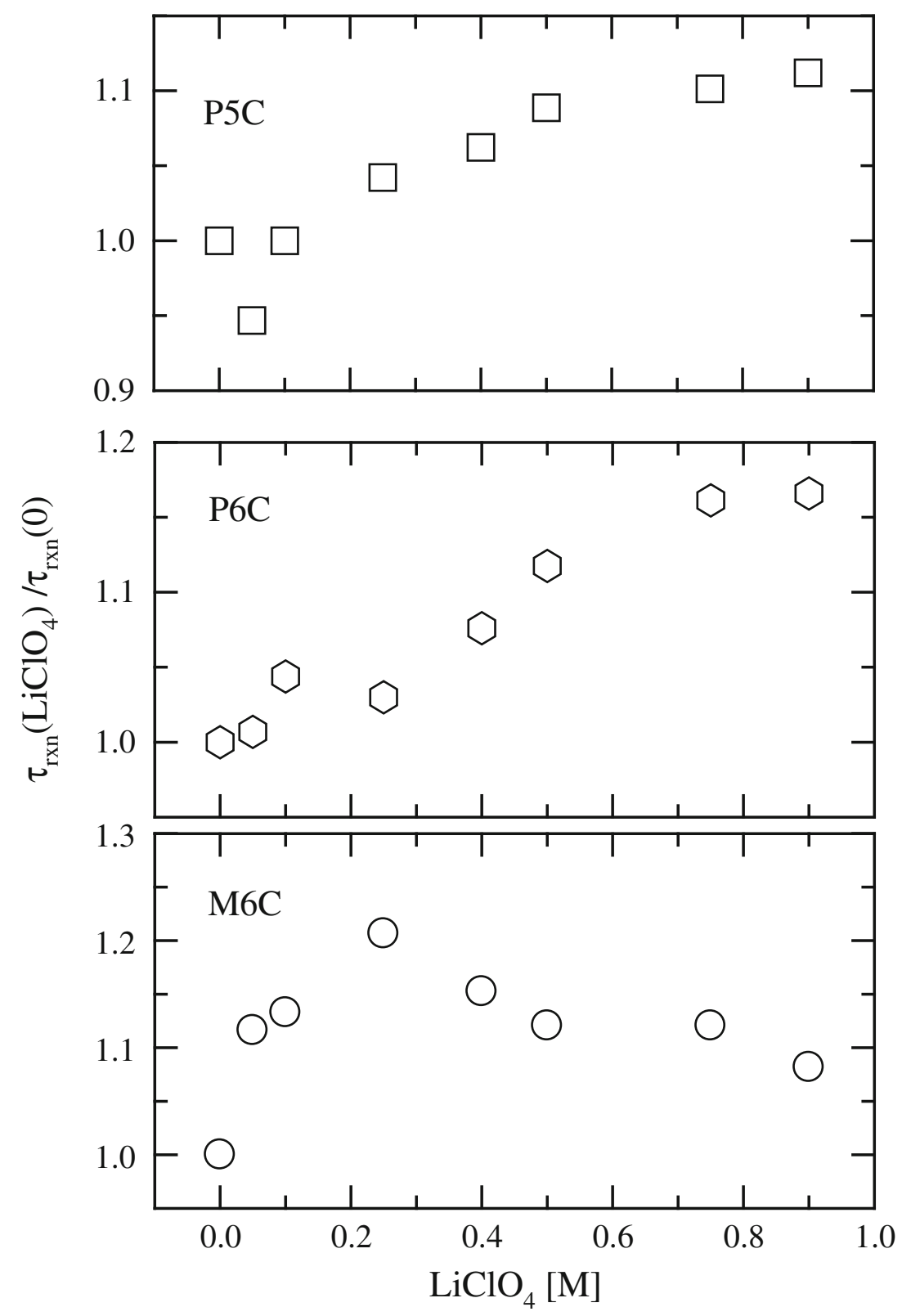

Figure 12. Electrolyte concentration dependence of reaction time constant in acetonitrile reverse micelles at a fixed $W_{s}$. Ratio between reaction time in the presence and absence of $\mathrm{LiClO}_{4}, \tau_{r x n}\left(\mathrm{LiClO}_{4}\right) / \tau_{r x n}(0)$, for the $\mathrm{LE} \rightarrow \mathrm{CT}$ conversion reaction of $\mathrm{P} 5 \mathrm{C}, \mathrm{P} 6 \mathrm{C}$ and $\mathrm{M} 6 \mathrm{C}$ in $\mathrm{AOT} / \mathrm{n}$-heptane/acetonitrile reverse micelles at $W_{s}=5$ are shown as a function of $\mathrm{LiClO}_{4}$ concentration. The squares, hexagons and circles represent data respectively for P5C, P6C and M6C. Error associated with the reaction time constants is typically $\pm 5 \%$ of the reported values. 
pool size, $W_{s}=5$. It is interesting to note that $\tau_{r x n}$ increases linearly with $\mathrm{LiClO}_{4}$ concentration for $\mathrm{P} 5 \mathrm{C}$ and $\mathrm{P} 6 \mathrm{C}$ but exhibits a non-monotonic dependence for M6C. Note a pronounced non-monotonic $\mathrm{LiClO}_{4}$ concentration dependence of $\tau_{r x n}$ was also observed earlier in bulk ethyl acetate with another closely related molecule of the PnC series. ${ }^{45}$ Data in figure 12 indicate $\tau_{r x n}$ increases only by $\sim 15 \%$ for P5C and $\sim 20 \%$ for $\mathrm{P} 6 \mathrm{C}$ at highest $\mathrm{LiClO}_{4}$ concentration over their respective values in the absence of any electrolyte at $W_{s}=5$. The peak value of $\tau_{r x n}$ for M6C is again $\sim 20 \%$ larger than that in the absence of $\mathrm{LiClO}_{4}$. This is surprising given the fact that the viscosity increases by nearly a factor of 2 in this concentration range (see figure 3). A much less lengthening of $\tau_{r x n}$ than expected on viscosity consideration probably suggests electrolyte-induced enhancement of solution polarity ${ }^{42}$ facilitating the ICT reaction in confined electrolyte solutions, leading to a partial decoupling from the local viscosity. This possibility, however, does not explain why the dependence of $\tau_{r x n}$ on electrolyte concentration shows a maximum for M6C but linear for other two reactants. The plots of $\ln \left[\tau_{r x n}^{-1}\right]$ versus the reaction driving force $\left(-\Delta G_{r}\right)$ do not indicate any different dependence for these three molecules and thus possibly rules out hidden role for reorganization energy $\left(\lambda_{s}\right)^{46}$ specific to any particular reactant among these three. The origin of different electrolyte concentration dependence of $\tau_{r x n}$ for M6C at this $W_{s}$ of AOT/heptane/acetonitrile reverse micelles are unknown to us at present and thus further studies are required to better understand this phenomenon.

\section{Conclusions}

The summary of the present work is as follows. The pool size dependence of $\mathrm{LE} \rightarrow \mathrm{CT}$ conversion reaction of photo-excited P5C, P6C and $\mathrm{M} 6 \mathrm{C}$ in $\mathrm{AOT} / \mathrm{n}-$ heptane/acetonitrile and AOT/n-heptane/methanol reverse micelles has been studied at room temperature. Absorption and fluorescence emission spectra of dissolved probe exhibit continuous red-shift with $W_{s}$, suggesting increase in polar solvent pool with it. Careful comparison of spectra taken at both smallest and largest $W_{s}$ values, and those in binary saturated solutions of polar and non-polar solvents reveals no partitioning of polar solvent and solute into the nonpolar phase of reverse micelles. In addition, formation of CT emission band shows gradual increase with $W_{s}$. Depending upon $W_{s}$, the formation of the more polar charge-transferred (CT) state in these confined environments has been found to be decreased by $10-100$ times compared to that observed in bulk solvents. The rate constant for this conversion reaction has also been found to be slowed down by 1-2 orders of magnitude. In order to correlate the observed equilibrium and time-dependent aspects of the ICT reaction with solvent properties, dielectric constant, viscosity and average solvation time have been estimated by following the fluorescence response of a non-reactive probe dissolved in these confined media. At $W_{s}=5$ for both acetonitrile and methanol, the estimated dielectric constants are found to be 3-5 times less than the bulk values. Slope of increase of dielectric constant with $W_{s}$ is steeper for confined methanol than that for confined acetonitrile but similar to that for confined water. The 'local' viscosity also increases by a factor of 2 at this $W_{s}$ for acetonitrile. Measured average solvation times are found to be at least three orders of magnitudes slower than those measured for bulk solvents. The reduction in rate constant by 1-2 orders of magnitude is argued to originate from the decreased solution polarity, enhanced local viscosity and slow solvent reorganization in these confined solvents.

Subsequently, electrolyte effects on an ICT reaction in confined environment, has been investigated at a fixed $W_{s}$ for acetonitrile reverse micelles. A maximum of $\sim 20 \%$ lengthening of the reaction time constant over the value in the absence of electrolyte $\left(\mathrm{LiClO}_{4}\right)$ has been observed in the concentration range of 0.0 $0.9 \mathrm{M}$. While the reaction time constant for P5C and $\mathrm{P} 6 \mathrm{C}$ increases linearly with electrolyte concentration in confined polar solvent, it shows a non-monotonic $\mathrm{LiClO}_{4}$ concentration dependence for M6C. Similar non-monotonic dependence is also observed for measured average solvation time but the estimated viscosity varies between 2 and 4 times of the bulk value. Much less reduction in reaction rate constant than expected from the viscosity consideration suggests more complex medium effects on ICT reaction in confined electrolyte solutions. The correlation between the reaction rate and reaction driving force has been found to be, on an average, linear for all these ICT molecules in both the presence and absence of electrolyte in this concentration range. We are, therefore, yet to understand the origin of non-monotonic electrolyte concentration dependence of ICT reaction rate constant in M6C.

The role of solute-medium interaction and medium dynamics can be further explored by following the reaction in binary liquid mixtures, molten non-aqueous electrolyte mixtures and solution $\mathrm{pH}$ dependence. In non-aqueous multi-component electrolyte mixtures ${ }^{96}$ one would like to investigate the common ion effects on equilibrium constant and time scale of an ICT 
reaction. Presence of proton in solution may influence the availability of the electron pair in the donor moiety for participation in the charge transfer process. Preferential solvation and diffusive time scale of solvent rearrangement in the first few solvation shells around a reactant may lead to more complex effects on ICT reaction than in neat solvents. Temperature dependent studies of ICT reaction in media, where heterogeneity rules the solution structure (for example, aqueous mixtures of tertiary butanol) may also provide important information about the role of solution structure on ICT reaction.

\section{Acknowledgements}

We thank Ms. S Sengupta and Dr. N Rohman for their help at the initial stage of this work. Financial support from the Council of Scientific and Industrial Resource (CSIR), India is gratefully acknowledged.

\section{Supporting information}

Tables S1-S3 and figures S1-S4 were provided as supplementary materials. See www.ias.ac.in/chemsci for supporting information.

\section{References}

1. Luisi P L and Straub B E (eds) 1984 Reverse micelles: Biological and technological relevance to amphiphilic structures in apolar media (Plenum: New York)

2. Levinger N E 2002 Science 2981722

3. Willard D M, Riter R E and Levinger N E 1998 J. Am. Chem. Soc. 120 4151; (b) Riter R E, Undiks E P and Levinger N E 1998 J. Am. Chem. Soc. 120 6062; (c) Riter R E, Willard D M and Levinger N E 1998 J. Phys. Chem. B 1022705

4. Harpham M R, Ladanyi B and Levinger N E $2005 \mathrm{~J}$. Phys. Chem. B 10916891

5. Menger F M, Donohue J A and Williams R F 1973 J. Am. Chem. Soc. 95 286; (b) Menger F M and Yamada K 1979 J. Am. Chem. Soc. 1016731

6. Bhattacharyya K 2003 Acc. Chem. Res. 36 95; (b) Bhattacharyya K and Bagchi B 2000 J. Phys. Chem. A 104 10603; (c) Ghosh S, Mandal U, Adhikari A, Dey S and Bhattacharyya K 2007 Int. Rev. Phys. Chem. 26421

7. Baruah B, Roden J M, Sedgwick M, Correa N M, Crans D C and Levinger N E $2006 \mathrm{~J}$. Am. Chem. Soc. 128 12758

8. De T and Maitra A 1995 Adv. Colloid Interface Sci. 59 95

9. Silber J J, Biasutti A, Abuin E and Lissi E $1999 A d v$. Colloid Interface Sci. 82189

10. Biswas R, Rohman N, Pradhan T and Buchner R 2008 J. Phys. Chem. B 1129379

11. Rico I and Lattes A 1984 J. Colloid Interface Sci. 102 285
12. Ray S and Moulik S P 1994 Langmuir 10, 2511

13. Hayes D G and Gulari E 1995 Langmuir 114695

14. Gautier M, Rico I, Samii A-Z, De Savignac A and Lattes A J 1986 J. Colloid Interface Sci. 112484

15. Mukherjee K, Moulik S P and Mukherjee D C 1993 Langmuir 91727

16. Mukherjee K, Mukherjee D C and Moulik S P 1994 J. Phys. Chem. 984713

17. Correa N M and Levinger N E 2006 J. Phys. Chem. B 11013050

18. Shirota H and Segawa H 2004 Langmuir 20329

19. Riter R E, Kimmel J R, Undiks E P and Levinger N E 1997 J. Phys. Chem. B 1018292

20. Falcone R D, Correa N M, Biasutti M A and Silber J J 2000 Langmuir 163070

21. Falcone R D, Silber J J and Correa N M 2009 Phys. Chem. Chem. Phys. 1111096

22. Novaira M, Moyano F, Biasutti M A, Silber J J and Correa N M 2008 Langmuir 24 4637; (b) El Seoud O A, Correa N M and Novaki L P 2001 Langmuir 17 1847; (c) Novaki L P, Correa N M, Silber J J and El Seoud O A 2000 Langmuir 165573

23. Mathew C, Saidi Z, Peyrelasse J and Boned C 1991 Phys. Rev. A 43873

24. Lopez-Cornejo P and Costa S M B 1998 Langmuir 14 2042

25. Laia C A T, Lopez-Cornejo P, Costa S M B, d'Oliveira J and Martinho J M G 1998 Langmuir 143531

26. Friberg S E, Rong G and Ward A J I 1988 J. Phys. Chem. 927247

27. Shchipunov Y A and Shumilina E V 1996 Colloid J. 58 123

28. Rico I and Lattes A 1987 In Microemulsion systems Rosano H L and Clausse M (eds) (New York: Marcel Dekker) Vol. 24

29. Mukherjee L, Mitra N, Bhattacharya P K and Moulik S P 1995 Langmuir 112866

30. Chhabra V, Lal M and Maitra A N 1995 J. Mater. Res. 102689

31. Singhal M, Chhabra V, Kang P and Shah D O 1997 Mater. Res. Bull. 32239

32. Venables D S, Huang K and Schmuttenmaer C A 2001 J. Phys. Chem. B 1059132

33. Setua P, Seth D and Sarkar N 2009 Phys. Chem. Chem. Phys. 118913

34. Shirota H and Horie K 1999 J. Phys. Chem. B 1031437

35. Hazra P, Chakrabarty D and Sarkar N 2002 Chem. Phys. Lett. 358523

36. Hazra P and Sarkar N 2002 Phys. Chem. Chem. Phys. 4 1040

37. Hazra P, Chakrabarty D and Sarkar N 2002 Langmuir 18 7872

38. Hazra P, Chakrabarty D and Sarkar N 2003 Chem. Phys. Lett. 371553

39. Binks B P, Kellay H and Meunier J 1991 Europhys. Lett. 1653

40. Yaghmur A, Asrein A, Antalek B and Gartri N 2003 Langmuir 191063

41. Tsao H K 1999 Langmuir 154981

42. Huppert D, Ittah V and Kosower E M 1989 Chem. Phys. Lett. 159267 
43. Novaira M, Biasutti M A, Silber J J and Correa N M 2007 J. Phys. Chem. B 111748

44. Pradhan T, Biswas R 2007 J. Phys. Chem. A 11111524

45. Pradhan T and Biswas R 2009 J. Solution Chem. 38 517

46. Pradhan T, Gazi H A R and Biswas R 2009 J. Chem. Phys. 131054507

47. Riddick J A, Bunger W B and Sakano T K 1986 Organic solvents (New York: Wiley)

48. Dahl K, Biswas R, Ito N and Maroncelli M 2005 J. Phys. Chem. B 1091563

49. Pradhan T, Gazi H A R and Biswas R 2010 J. Chem. Sci. 122 481; (b) Gazi H A R and Biswas R 2011 J. Chem. Sci. 123265

50. Grabowski Z R, Rotkiewicz K and Rettig W 2003 Chem. Rev. 1033899

51. Lippert E, Rettig W, Bonacic-Koutecky V, Heisel F and Mieche J A 1987 Adv. Chem. Phys. 681

52. Hicks J, Vandersall M, Babarogic Z and Eisenthal K B 1985 Chem. Phys. Lett. 11618

53. Hicks J, Vandersall M T, Sitzmann E V and Eisenthal K B 1987 Chem. Phys. Lett. 135413

54. Nag A, Kundu T and Bhattacharyya K 1989 Chem. Phys. Lett. 160257

55. Horng M L, Gardecki J A, Papazyan A and Maroncelli M 1995 J. Phys. Chem. 9917311

56. Biswas R, Lewis J E and Maroncelli M 1999 Chem. Phys. Lett. 310485

57. Reynolds R, Gardecki J A, Frankland S J V, Horng M L and Maroncelli M 1996 J. Phys. Chem. 10010337

58. Hynes J T 1994 Charge-transfer reactions and solvation dynamics in ultrafast dynamics of chemical systems Simon J D (ed) (Dodrecht: Kluwer) p. 345

59. van der Zwan G and Hynes J T 1991 Chem. Phys. 152 169

60. Krishna M M G 1999 J. Phys. Chem. A 1033589

61. Rettig W J 1980 J. Lumin. 2621

62. Rettig W 1982 J. Phys. Chem. 861970

63. Zachariasse K A, Grobys M, von der Haar T, Hebecker A, Il'ichev Y V, Jiang Y-B, Morawski O and Kuhnle W 1996 J. Photochem. Photobiol. A 10259

64. Zachariasse K A 2000 Chem. Phys. Lett. 3208

65. Zachariasse K A, Druzhinin S I, Bosch W and Machinek R 2004 J. Am. Chem. Soc. 1261705

66. Techert S and Zachariasse K A 2004 J. Am. Chem. Soc. 1265593

67. Pradhan T and Biswas R 2007 J. Phys. Chem. A 111 11514

68. Pradhan T, Ghoshal P and Biswas R 2008 J. Phys. Chem. A 112915

69. Pradhan T, Ghoshal P and Biswas R 2009 J. Chem. Sci. 12195

70. Lewis J E, Biswas R, Robinson A G and Maroncelli M 2001 J. Phys. Chem. B $\mathbf{1 0 5} 3306$

71. Lakowicz J R 1999 Principles of fluorescence spectroscopy, 2nd edn (New York: Kluwer Academic/ Plenum Publishers)
72. Maroncelli M and Fleming G R 1987 J. Chem. Phys. 86 6221

73. Chapman C F and Maroncelli M 1991 J. Phys. Chem. 95 9095

74. Horng M L, Gardecki J A and Maroncelli M 1997 J. Phys. Chem. A $\mathbf{1 0 1} 1030$

75. Zhu D-M, Wu X and Schelly Z A 1992 J. Phys. Chem. 967121

76. Senapati S and Chandra A 2001 J. Phys. Chem. B 105 5106

77. Senapati S and Chandra A 1999 J. Phys. Chem. B 111 1223

78. Gray C G and Gubbins K E 1984 Theory of molecular fluids (Oxford: Clarendon) Vol. I

79. Hansen J P and McDonald I R 1976 Theory of simple liquids (Academic Press)

80. Ghosh S, Ramanathan K V and Sood A K 2004 Europhys. Lett. 65678

81. Koga K, Gao G T, Tanaka H and Zeng X C 2001 Nature 412 802; (b) Koga K, Gao G T, Tanaka H and Zeng X C 2002 Physica $\mathbf{3 1 4} 462$

82. Marti J and Gordillo M C 2001 Phys. Rev. E 64021504 ; (b) Gordillo M C and Marti J 2000 Chem. Phys. Lett. 329341

83. Sansom M S P and Biggin P C 2001 Nature 414156

84. Hummer G, Rasiah J C and Noworyta J P 2001 Nature 414188

85. Sando G M, Dahl K and Owrutsky J C 2005 J. Phys. Chem. B 1094084

86. Biswas R, Das A R, Pradhan T, Touraud D, Kunz W and Mahiuddin S 2008 J. Phys. Chem. B 1126620

87. Bagchi B and Biswas R 1999 Adv. Chem. Phys. 109207

88. Fee R S and Maroncelli M 1994 Chem. Phys. 183235

89 . Note that the above correlation, which has been constructed from the room temperature liquid data, may not quantitatively represent the viscosity dependence of solute rotation in spatially heterogeneous media such as those inside reverse micelles. In the absence of any simple yet better description, the same correlation has been used to estimate local viscosity experienced by a probe trapped in polar solvent pool.

90. Biswas R and Bagchi B 1997 J. Chem. Phys. 1065587

91. Hartman R S, Konitsky W M and Waldeck D H 1993 J. Am. Chem. Soc. 1159692

92. Kumar P V and Maroncelli M 2000 J. Chem. Phys. 112 5370

93. Kurnikova M G, Balabai N, Waldeck D H and Coalson R D 1998 J. Am. Chem. Soc. 1206121

94. Kurnikova M G and Waldeck D H 1996 J. Chem. Phys. 105628

95. Changes in 'local viscosity' borne out by the rotation data summarized in references 34 and 35 become more quantitative if the initial anisotropy $\mathrm{r}(0)$ values are fixed.

96. Guchhait B, Gazi H A R, Kashyap H K and Biswas R 2010 J. Phys. Chem. B 114 5066; (b) Gazi H A R, Guchhait B, Daschakraborty S and Biswas R 2011 Chem. Phys. Lett. 501358 